\title{
Pharmacokinetics of caffeine: A systematic analysis of reported data for application in metabolic phenotyping and liver function testing
}

\author{
Jan Grzegorzewski ${ }^{1}$, Florian Bartsch ${ }^{1}$, Adrian Köller ${ }^{1}$, and Matthias König ${ }^{1, *}$ \\ ${ }^{1}$ Institute for Theoretical Biology, Humboldt-University Berlin, Invalidenstraße 110, \\ 10115 Berlin, Germany
}

Correspondence*:

Matthias König

koenigmx@hu-berlin.de

\section{ABSTRACT}

Caffeine is by far the most ubiquitous psychostimulant worldwide found in tea, coffee, cocoa, energy drinks, and many other beverages and food. Caffeine is almost exclusively metabolized in the liver by the cytochrome P-450 enzyme system to the main product paraxanthine and the additional products theobromine and theophylline. Besides its stimulating properties, two important applications of caffeine are metabolic phenotyping of cytochrome P450 1A2 (CYP1A2) and liver function testing. An open challenge in this context is to identify underlying causes of the large inter-individual variability in caffeine pharmacokinetics. Data is urgently needed to understand and quantify confounding factors such as lifestyle (e.g. smoking), the effects of drug-caffeine interactions (e.g. medication metabolized via CYP1A2), and the effect of disease. Here we report the first integrative and systematic analysis of data on caffeine pharmacokinetics from 148 publications and provide a comprehensive high-quality data set on the pharmacokinetics of caffeine, caffeine metabolites, and their metabolic ratios in human adults. The data set is enriched by meta-data on the characteristics of studied patient cohorts and subjects (e.g. age, body weight, smoking status, health status), the applied interventions (e.g. dosing, substance, route of application), measured pharmacokinetic time-courses, and pharmacokinetic parameters (e.g. clearance, half-life, area under the curve). We demonstrate via multiple applications how the data set can be used to solidify existing knowledge and gain new insights relevant for metabolic phenotyping and liver function testing based on caffeine. Specifically, we analyzed (i) the alteration of caffeine pharmacokinetics with smoking and use of oral contraceptives; (ii) drug-drug interactions with caffeine as possible confounding factors of caffeine pharmacokinetics or source of adverse effects; (iii) alteration of caffeine pharmacokinetics in disease; and (iv) the applicability of caffeine as a salivary test substance by comparison of plasma and saliva data. In conclusion, our data set and analyses provide important resources which could enable more accurate caffeine-based metabolic phenotyping and liver function testing.

Keywords: caffeine, pharmacokinetics, smoking, oral contraceptives, drug-drug interactions, drug-disease interactions, CYP1A2, liver function test 


\section{INTRODUCTION}

Caffeine is commonly found in tea, coffee, cocoa, energy drinks, and many other beverages. It is by far the most ubiquitous psychostimulant worldwide (Gilbert, 1984), with $85 \%$ of the U.S. population consuming caffeine daily (Mitchell et al., 2014). Among caffeine consumers, the average consumption is more than $200 \mathrm{mg}$ of caffeine per day (Frary et al., 2005). Caffeine is mainly known for its stimulating properties but is also consumed for improved exercise performance and the treatment of various diseases (e.g. apnea in prematurity, hypersomnia). Two important applications of caffeine are liver function testing and metabolic phenotyping of cytochrome P450 1A2 (CYP1A2), N-acetyltransferase 2 (NA2), and xanthine oxidase (XO) (Wang et al., 1985; Jost et al., 1987; Wahlländer et al., 1990; Tripathi et al., 2015).

Caffeine is almost exclusively metabolized in the liver by the cytochrome P450 enzyme system with $3 \%$ or less being excreted unchanged in urine (Kot and Daniel, 2008). In humans, N-3 demethylation of caffeine (1,3,7-trimethylxanthine) to paraxanthine (1,7-dimethylxanthine) is the main reaction in the metabolism of caffeine, accounting for around $80-90 \%$ of caffeine demethylation. The reaction is exclusively mediated by the activity of the cytochrome P450 isoform 1A2 (CYP1A2) (Hakooz, 2009). The remainder of caffeine is metabolized to around $11 \%$ and $4 \%$ to the 1-demethylated product theobromine and 7-demethylated product theophylline, respectively (Lelo et al., 1986b; Kalow and Tang, 1993; Miners and Birkett, 1996; Amchin et al., 1999).

Large variation exists in the consumption of caffeine-containing beverages and food between individuals, which can induce CYP1A2 activity. In addition, CYP1A2 activity and protein amount are affected by environmental, genetic, and epigenetic factors (Klein et al., 2010) resulting in large variation between 5-6 fold in humans (Schrenk et al., 1998). These factors lead to a wide range of caffeine plasma concentrations and caffeine pharmacokinetics.

Sex does not significantly influence the CYP1A2 activity (Klein et al., 2010; Yang et al., 2010; Puri et al. 2020). A large heritability of CYP1A2 activity could be shown by a twin study (Rasmussen et al., 2002). However, satisfying genetic or epigenetic markers- on the CYP1A locus on chromosome 15 could not be found by follow-up studies (Jiang et al., 2006; Ghotbi et al., 2007; Gunes and Dahl, 2008; Klein et al., 2010; Yang et al., 2010). Other genes, regulating the expression and function of CYP1A2 and nongenetic factors could explain $42 \%, 38 \%$, and $33 \%$ of CYP1A2 variation at activity, protein, and mRNA level, respectively (Klein et al., 2010). Lifestyle factors (e.g. smoking) and use of oral contraceptives have been shown to influence caffeine pharmacokinetics, as have pregnancy, obesity, alcohol consumption, and the coadministrations of drugs (e.g. fluvoxamine and pipmedic acid). Many diseases reduce the metabolic capabilities of patients. For caffeine which is predominantly metabolized by the liver, various liver diseases result in a strong reduction in caffeine clearance. The most profound reduction is observed in cirrhotic liver disease, correlating with the degree of hepatic impairment (Holstege et al., 1989; Park et al., 2003; Jodynis-Liebert et al., 2004; Tripathi et al., 2015).

Metabolic phenotyping of enzymes by probe drugs is a common method to evaluate the impact of lifestyle, drug-gene and drug-drug interactions, and other factors influencing enzyme activity. Caffeine is an established probe drug for CYP1A2, N-acetyltransferase 2 (NAT2), and xanthine oxidase (XO) metabolic activities (Miners and Birkett, 1996; Fuhr et al., 1996; Faber et al., 2005; Hakooz, 2009). It is rapidly and completely absorbed by the gastrointestinal tract, distributed throughout the total body water, has low plasma binding, as well as short half-life, negligible first-pass metabolism (Yesair et al., 1984), minimal renal elimination, excellent tolerability, and its biotransformation is virtually confined to the liver (Amchin et al., 1999; Kalow and Tang, 1993; Drozdzik et al., 2018). Caffeine is especially used for CYP1A2 phenotyping which contributes $5-20 \%$ to the hepatic $\mathrm{P} 450$ pool and is involved in the clearance 
of about $9 \%$ of clinically used drugs (Zanger and Schwab, 2013). The partial or systemic caffeine clearance measured in plasma is considered to be the gold standard for CYP1A2 phenotyping (Fuhr et al. 1996) since $95 \%$ of the systemic clearance of caffeine is estimated to be due to hepatic CYP1A2 (Amchin et al., 1999). Measurements in serum, saliva, and urine are extensively studied as well. In urine, sampling at multiple time points and precise timing are inherently difficult. Thus, clearance rates are typically calculated only from plasma, saliva, and serum samples. Measurements in saliva are not invasive and show good correlation with measurements in plasma (Callahan et al., 1982; Wahlländer et al., 1990). The metabolic ratio (MR) between various metabolites of caffeine is an established alternative measure for CYP1A2 enzyme activity (Hakooz, 2009). Analogously, the MR is measured in any of the above mentioned tissues though typically only at a single time point after drug administration. The MR of various metabolites at 4 hours after dosing in plasma, saliva, and urine correlate well with the apparent caffeine clearance, 0.84, 0.82, 0.61, respectively (Carrillo et al., 2000). The MRs measured in plasma and urine have been historically most popular. However, measurements in saliva are routinely applied, especially in epidemiological studies (Tantcheva-Poór et al., 1999; Kukongviriyapan et al., 2004; Tripathi et al. 2015; Chia et al., 2016; Urry et al., 2016; Puri et al., 2020).

Despite the great potential of caffeine as a test substance for liver function tests and CYP1A2 based phenotyping, so far caffeine testing has not found widespread clinical adoption. For liver function tests, a major limiting factor is the large inter-individual variability. Data is urgently needed to understand and quantify confounding factors of caffeine pharmacokinetics such as lifestyle (e.g. smoking) and the effects of drug-drug interactions (e.g. drugs metabolized via CYP1A2) or how disease alters caffeine elimination. Based on such data, more accurate liver function tests and CYP1A2 phenotyping protocols could be established. Differences in clinical protocols (e.g. dosing amount, sampling tissue, and timing) have not been systematically analyzed in the literature. In addition, data on competing substances in the context of dynamical liver function tests (e.g. metacethin used in the LiMAx test (Rubin et al., 2017)) and CYP1A2 phenotyping is not accessible but absolutely imperative for a quantitative evaluation of these methods.

Caffeine pharmacokinetics have been investigated in a multitude of clinical trials, each with its own focus and research question. These studies have been reviewed in a broad scope, most recently in (Arnaud, 2011; Nehlig, 2018). Despite caffeine pharmacokinetics being highly studied in literature, no integrated pharmacokinetics data set exists so far and no systematic analysis of the reported data has been performed. The objective of this work was to fill this gap by providing the first comprehensive high-quality data set of reported data on caffeine pharmacokinetics and demonstrate its value via multiple example applications relevant for metabolic phenotyping and liver function testing based on caffeine.

\section{MATERIAL AND METHODS}

\subsection{Data curation}

Publications containing caffeine pharmacokinetics data were searched on PubMed and Google Scholar using combinations of keywords related to caffeine and pharmacokinetics. Most publications were retrieved by combining the keyword caffeine with one of the following keywords: pharmacokinetics, pk, plasma concentration, serum concentration, saliva, time profile, time-course, concentration-time profile, clearance, half-life, elimination rate, or area under the curve (AUC). Based on this initial corpus, additional publications were added by following references and citations. Pharmacokinetics data was curated manually as part of the pharmacokinetics database PK-DB (https://pk-db.com/) (Grzegorzewski et al. 2020) using established workflows. The pharmacokinetics data was stored in combination with 
relevant metadata on groups, individuals, interventions, and outputs. PK-DB provided support in the curation process with strong validation rules, unit normalization, and automatic calculation of pharmacokinetic parameters from time-courses. As part of the curation process and the presented analyses, pharmacokinetic parameters and other commonly reported measurements were integrated from multiple studies. The meta-analyses and data integration allowed to identify and correct/remove outlier data which were mostly due to either curation errors or incorrect reporting. Pharmacokinetic parameters calculated from time-courses are included in the analyses. For more details see (Grzegorzewski et al., 2020).

\subsection{Data processing and filtering}

Certain data processing and data filtering methods were applied in all analyses. Data included in the analyses was either measured in plasma, serum, or saliva (urinary measurements were excluded). The data was neither filtered based on application route (e.g. oral, intravenous, intramuscular) nor application form (e.g. tablet, capsule, solution) of caffeine. Male subjects were assumed not to take any oral contraceptives. Data outliers from four studies (Stille et al., 1987; Harder et al., 1988, 1989; Balogh et al., 1992) were excluded from all analyses. All four studies probably originate from the same clinical investigation which was detected as outliers during the data curation process.

In Fig. 2A, B and Fig. 3A-D, the data is displayed in a similar manner. For collectively reported subjects, the group size and standard error is displayed as the marker size and error-bar, respectively. In the legend, (I), (G), and (TI) stand for individual participant data, the number of groups, and the total number of subjects, respectively. Data points are depicted as circles if reported equivalently in the source, as squares if calculated from concentration-time profiles and as triangles if inferred from corresponding pharmacokinetic data and body weights of the subjects. Typically, dosing is reported in mass units, AUC in mass per volume units, clearance in volume per time units, and half-life in time units. Occasionally, dosage, AUC, and clearance are reported in body weight units. In case of reported subject weight, the data is harmonized to similar units by multiplying with the reported weights.

In Fig. 2, the depicted subjects were healthy. Substances with negligible caffeine-drug interactions were determined by an effect size analysis in Sec. 3.4. All other co-administrations and investigations containing multiple caffeine dosages were excluded.

In subplot Fig. $3 \mathrm{~A}$, included subjects were healthy. The area under the caffeine concentration curves measured at least up to 12 hours after a single application of caffeine and AUC extrapolated to infinity were included. Multiple subsequent caffeine dosages were excluded, other administrations and coadministrations included. In subplot Fig. 3 $\mathrm{C}$, healthy and non-healthy subjects were included. Single applications of caffeine or caffeine administrated as a cocktail with negligible drug-drug interactions were included. All other co-administrations and investigations containing multiple caffeine dosages were excluded.

In subplots Fig. 4A-C, no data was excluded. In subplot Fig. 4D, included subjects were healthy, nonsmoking, non-pregnant, and non-oral contraceptive consumers. Interventions with caffeine administrated as a cocktail with negligible caffeine-drug interactions were included. All other co-administrations and investigations containing multiple caffeine dosages were excluded.

\section{RESULTS}

\subsection{Caffeine pharmacokinetics data set}


bioRxiv preprint doi: https://doi.org/10.1101/2021.07.12.452094; this version posted August 3, 2021. The copyright holder for this preprint (which was not certified by peer review) is the author/funder. All rights reserved. No reuse allowed without permission.

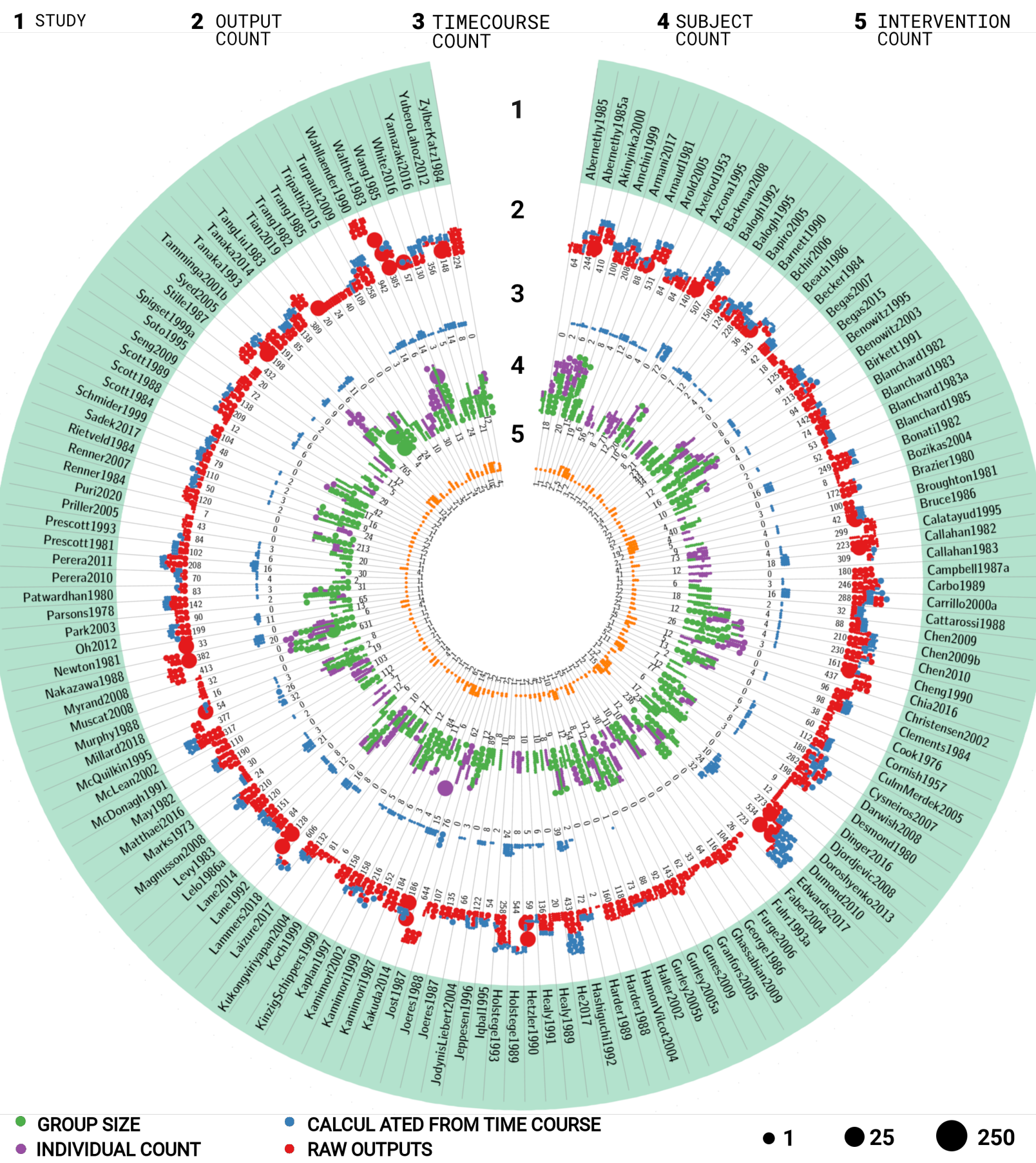

Figure 1. Overview of studies in the caffeine pharmacokinetics data set. The data set consists of 148 studies containing 1311 groups, 12106 individuals, 1650 interventions, 97026 outputs, and 4224 timecourses. The circular plot is structured in stripes and rings. Each stripe represents a different study, each ring the amount of different data types for the respective study. The dots represent the respective amount of data with the dot size corresponding to the number of entries per dot. The rings contain the following information for the respective study (1) name of the study; (2) number of outputs (pharmacokinetics parameters and other measurements). Red dots represent reported data, blue dots data calculated from time-courses reported in the study; (3) number of time-courses; (4) number of participants. Purple dots represent participants with individual data, green dots represent collectively reported participants; (5) number of interventions applied to the participants in the study. For additional information see Tab. 1. 
Abernethy and Todd 1985, Akinvinka et al. 2000$)$ Amchin et al. 1999 Armani et al. 2017) Arnaud and Welsch 1982 Axelrod and Reichenthal 1953) Azcona et al. 1995) Backman et al. 2008 Balogh et al. 1992 Balogh et al. 1995 Bapiro et al. 2005 Barnett et al. 1990

Bchir et al. 2006)

Becker et al. 1984

Begas et al. 2007)

Begas et al. 2015

Benowitz et al. 1995 Benowitz et al. |2003. Blanchard and Sawers 1983b

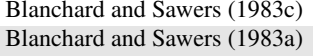
\begin{tabular}{ll}
\hline Blanchard et al. 1985 \\
\hline Bonati et al 1982
\end{tabular}

Bonati et al. 1982)

\begin{tabular}{|l|}
\hline Bozikas et al. 2004 \\
\hline Brazier et al. 11980 \\
\hline
\end{tabular}

\begin{tabular}{l|l} 
Broughton and Rogers & $1981)$ \\
\hline
\end{tabular}

Bruce et al. 1986)

Calatayud et al. (1995)

If

PKDB00427

PKDB00002 PKDB00003

PKDB00428

PKDB00429

\begin{tabular}{l} 
PKDB00430 \\
\hline PKDB00431
\end{tabular}

PKDB00432

\begin{tabular}{l} 
PKDB00004 \\
\hline PKDB00005 \\
\hline FKDB0006
\end{tabular}

PKDB00005

PKDB00006

PKDB00433

\begin{tabular}{l|l|} 
PKDB00008 \\
\hline PKDB00435
\end{tabular}

\begin{tabular}{r|r|} 
PKDB00435 \\
\hline PKDB00009
\end{tabular}

\begin{tabular}{l|l} 
PKDB00009 \\
\hline PKDB00436
\end{tabular}

\begin{tabular}{|r|r|}
\hline PKDB00010 & 12 \\
\hline PKDBO0011 & 12
\end{tabular}

PKDBO0011

PKDB00012

PKDB00210

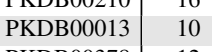

PKDB00378

PKDB00437

PKDB00438

PKDB00439 $\quad$\begin{tabular}{rr}
4 & 0 \\
\hline & 0
\end{tabular}

\begin{tabular}{r|rr|r}
\hline PKDB00441 & 9 & 1 & $\varnothing$ \\
\hline PKDBO042 & 2 &
\end{tabular}

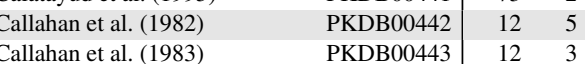

\begin{tabular}{ll|l} 
& PKDB00443 & 12 \\
\hline
\end{tabular}

Campbell et al. (1987)
Carbó et al. (1989)

\begin{tabular}{|r|r|r|}
\hline PKDB00015 & 15 \\
\hline & 15
\end{tabular}

PKDB00016

\begin{tabular}{|l|l|l|}
\hline PKDB 00017 & 23 \\
\hline PKDBO044 & & 4 \\
\hline
\end{tabular}

\begin{tabular}{|l|}
\hline PKDBO0444 \\
\hline PKDBO0445
\end{tabular}

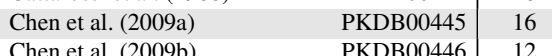

\begin{tabular}{ll|ll|l}
\hline Chen et al. 2009b & PKDB00446 & 12 & 1 & $\checkmark$
\end{tabular}

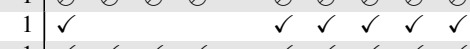

\begin{tabular}{|l|l|l|l|}
\hline Cheng et al. (1990) & PKDB00018 & 26 & 3 \\
\hline Chia et al. 2016
\end{tabular}

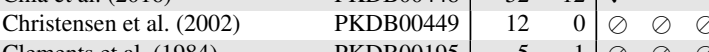

Clements et al. 1984

\begin{tabular}{r|rr}
\hline PKDBD0195 & 5 & 1
\end{tabular}

Cornish and Christman 1957

Culm-Merdek et al. (2005,

Darwish et al. 2008)

PKDBO0019

PKDB00451

PKDB00328

\begin{tabular}{ll|lll}
7 & 1 & $\oslash$ & $\oslash$ & $\varnothing$
\end{tabular}

o)
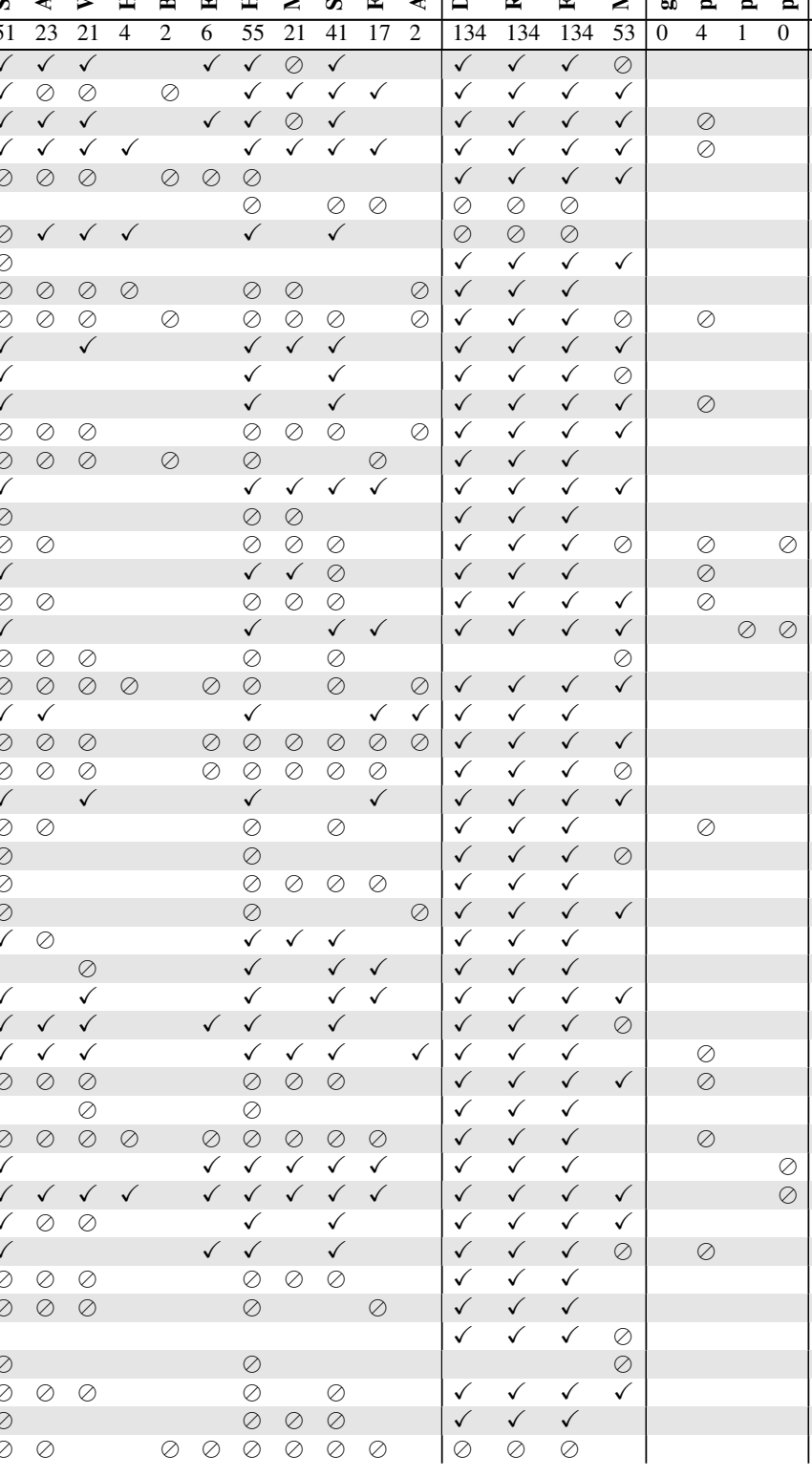

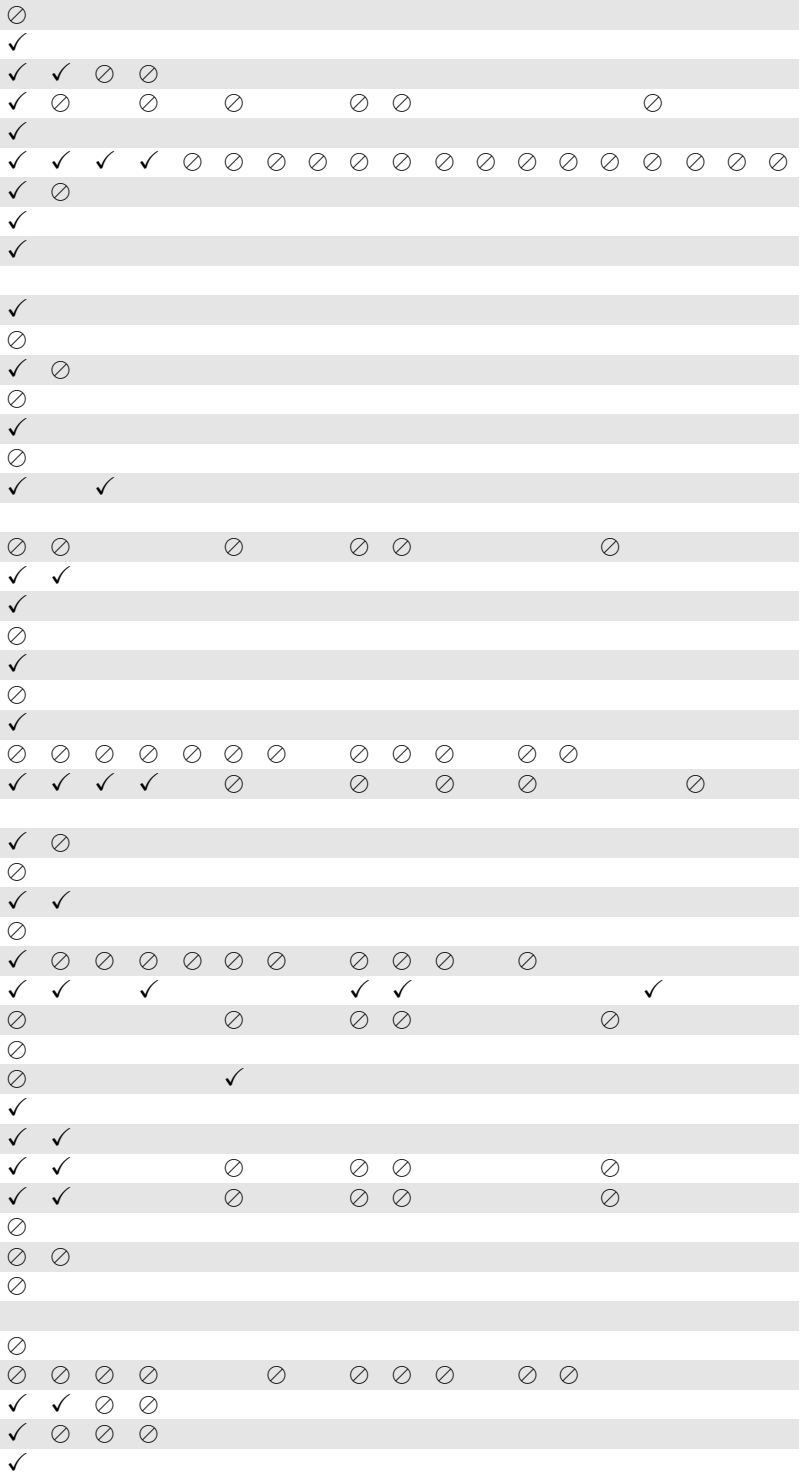

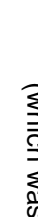

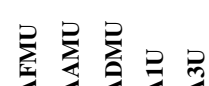




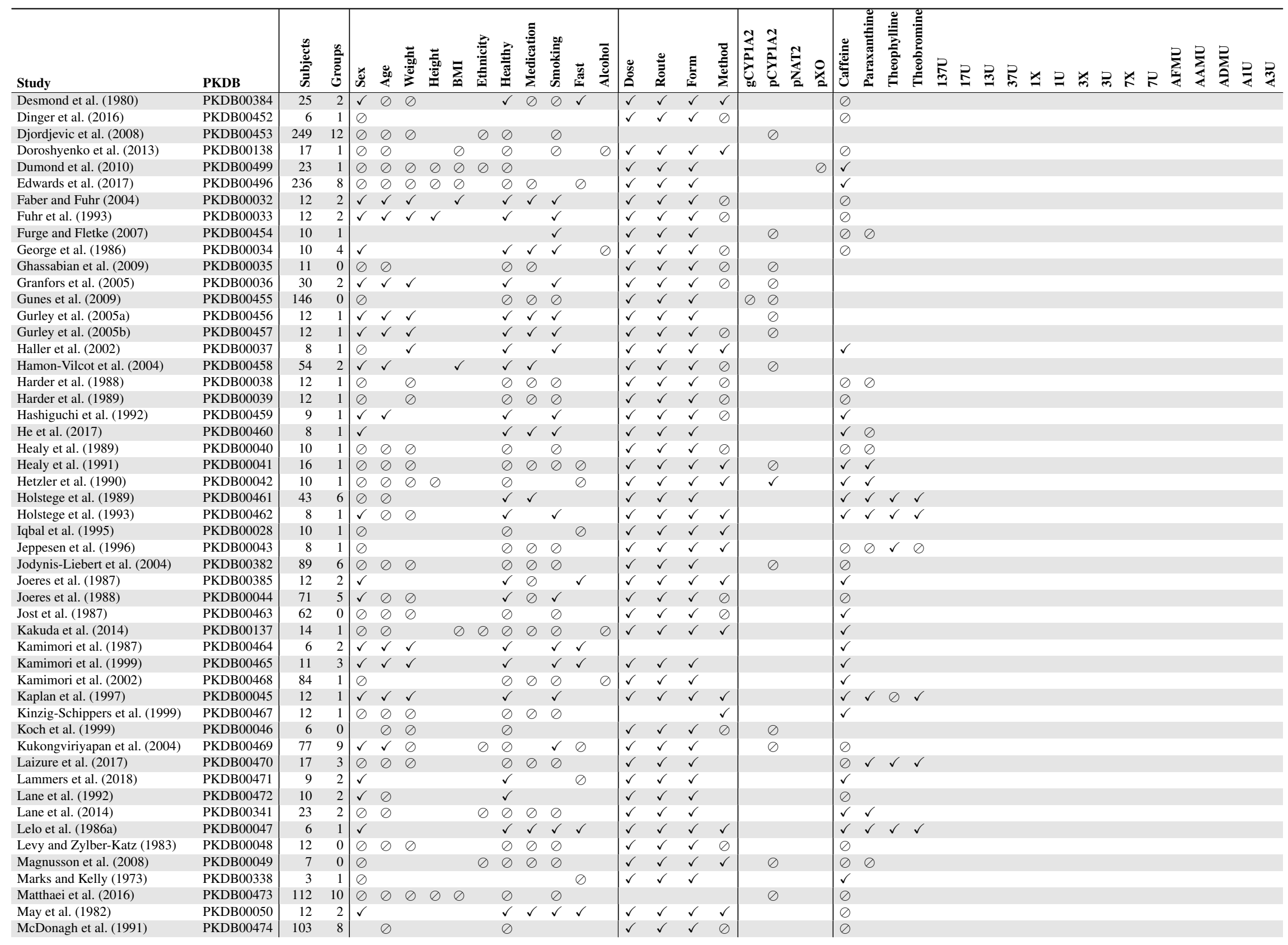


Study PKDB

\section{Hin:min an}

McLean and Graham 2002)

McQuilkin et al. 1995

Millard et al. 2018)

Murphy et al. 1988

\begin{tabular}{|l|l|}
\hline Muscat et al. & 2008) \\
\hline Myrand et al & 2008 \\
\hline
\end{tabular}

Nakazawa and Tanaka 1988)

Newton et al. 1981

\begin{tabular}{l} 
Oh et al. 2012) \\
\hline Park et al. 2003)
\end{tabular}

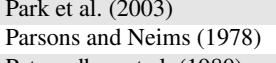

Patwardhan et al. 1980)

Perera et al. 2010)

Prescott et al. 1981

Prescott et al. 1993

\begin{tabular}{l} 
Priller 2005) \\
\hline Puri et al. 2020
\end{tabular}

Renner et al. 1984

Renner et al. 2007)

\begin{tabular}{l} 
Rietveld et al. 1984 \\
\hline Sadek et al. 12017)
\end{tabular}

Schmider et al. 1999

Scott et al. 1984)

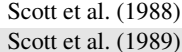

Seng et al. 2009)

Soto et al. 1995)

Stille et al. (1987)

Syed et al. 2005)

Tamminga et al. 200

\begin{tabular}{l|l|}
\hline Tanaka et al. & 1993 \\
\hline Tanaka et al. & 2014 \\
\hline
\end{tabular}

Tang-Liu et al. 1983

Tian et al. 2019)

Trang et al. 1982)

$\frac{\text { Trang et al. 1985) }}{\text { Tripathi et al. |2015) }}$

Turpault et al. |2009)

Wahlländer et al. 1990

Wang et al. 1985

Wang et al. 1985,

\begin{tabular}{|l|}
\hline White et al. |2016 \\
\hline Yamazaki et al. 2017 \\
\hline
\end{tabular}

Yamazaki et al. 2017)

\begin{tabular}{l} 
Yubero-Lahoz et al. 201 \\
\hline Zylber-Katz et al. 1984 \\
\hline
\end{tabular}

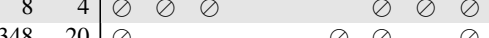

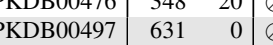

\begin{tabular}{r|rr|lllllll} 
& 12 & 1 & 0 & 0 & 0 & 0 & 0 & 0 & 0
\end{tabular}

\begin{tabular}{l|cc|ccc} 
PKDBO0053 & 6 & 1 & $\varnothing$ & $\oslash$ & $\varnothing$
\end{tabular}

\begin{tabular}{l|ll|}
\hline PKDB00054 & 13 & 1
\end{tabular}

0000

PKDB00057

\begin{tabular}{ll|ll}
26 & 2 & $\checkmark$ & $\checkmark$
\end{tabular}

PKDB00058

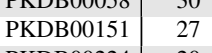

\begin{tabular}{|l|l|l|}
\hline PKDB00224 & 20 & 3 \\
\hline PKDB00479 & 64 & 11 \\
\hline
\end{tabular}

\begin{tabular}{|r|rr|}
\hline PKDB00479 & 64 & 11 \\
\hline PKDB00480 & 213 \\
\hline
\end{tabular}

PKDB00059

PKDBO0125

\begin{tabular}{|r|r|r|r|}
\hline PKDB00481 & 9 & 0 \\
\hline & &
\end{tabular}

\begin{tabular}{|l|ll|}
\hline PKDB00482 & 16 & 2 \\
\hline PKDB00483 & 17 & 1 \\
\hline
\end{tabular}

\begin{tabular}{|l|ll|}
\hline PKDB00483 & 17 & 1 \\
\hline PKDB00484 & 42 & 0 \\
\hline PKDBO0455 & 24 & 3 \\
\hline
\end{tabular}

\begin{tabular}{l|l|l|l|l|}
\hline PKDB00484 & 42 \\
\hline PKDB00485 & 24 \\
\hline
\end{tabular}

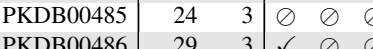

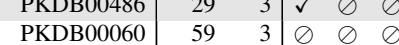

\begin{tabular}{r|rr|rrr} 
PKDB 00487 & 5 & 1 & $\checkmark$ & $\checkmark$ & $\checkmark$
\end{tabular}

\begin{tabular}{|r|rr|r|}
\hline PKDB00061 & 12 & 1 & 8 \\
\hline PKDB00062 & 12 & 0 \\
\hline PKDBO068 & 48 & 3 & 0
\end{tabular}

\begin{tabular}{r|rr|r} 
PKKDB00488 & 48 & 3 & $\oslash$ \\
\cline { 1 - 3 } & 765 & 0 & $\oslash$
\end{tabular}

\begin{tabular}{r|rr|r} 
PKDB00498 & 765 & 0 & $\oslash$ \\
\hline PKDB00489 & 10 & 1 & $\varnothing$
\end{tabular}

\begin{tabular}{|l|ll|l}
\hline PKDB00136 & 4 & 1 & $\oslash$ \\
\hline PKDB00126 & 6 & 1 & $\checkmark$
\end{tabular}

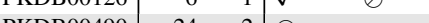

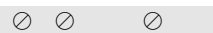

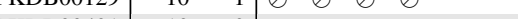

\begin{tabular}{|l|ll|l}
\hline PKDBO0491 & 10 & 0 & $\varnothing$
\end{tabular}

\begin{tabular}{l|ll|l}
\hline PKDBOO492 & 45 & 5 & $\checkmark$ \\
\hline
\end{tabular}

\begin{tabular}{|r|rr|r|r|}
\hline PKDDB0063 & 30 & 1 & $\oslash$ \\
\hline PKD & 10 & $\checkmark$ \\
\hline
\end{tabular}

\begin{tabular}{lllll}
\hline & 0
\end{tabular}

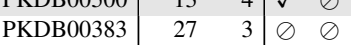

$\begin{array}{lllllllllll}24 & 1 & \theta & 0 & 0 & 0 & 0 & \theta & \theta & \theta\end{array}$

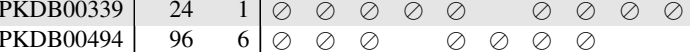

PKDB00065

\begin{tabular}{ll|lll}
21 & 2 & $\oslash$ & 0 & 0 \\
12 & 0 & 0 & 0 & 0
\end{tabular}
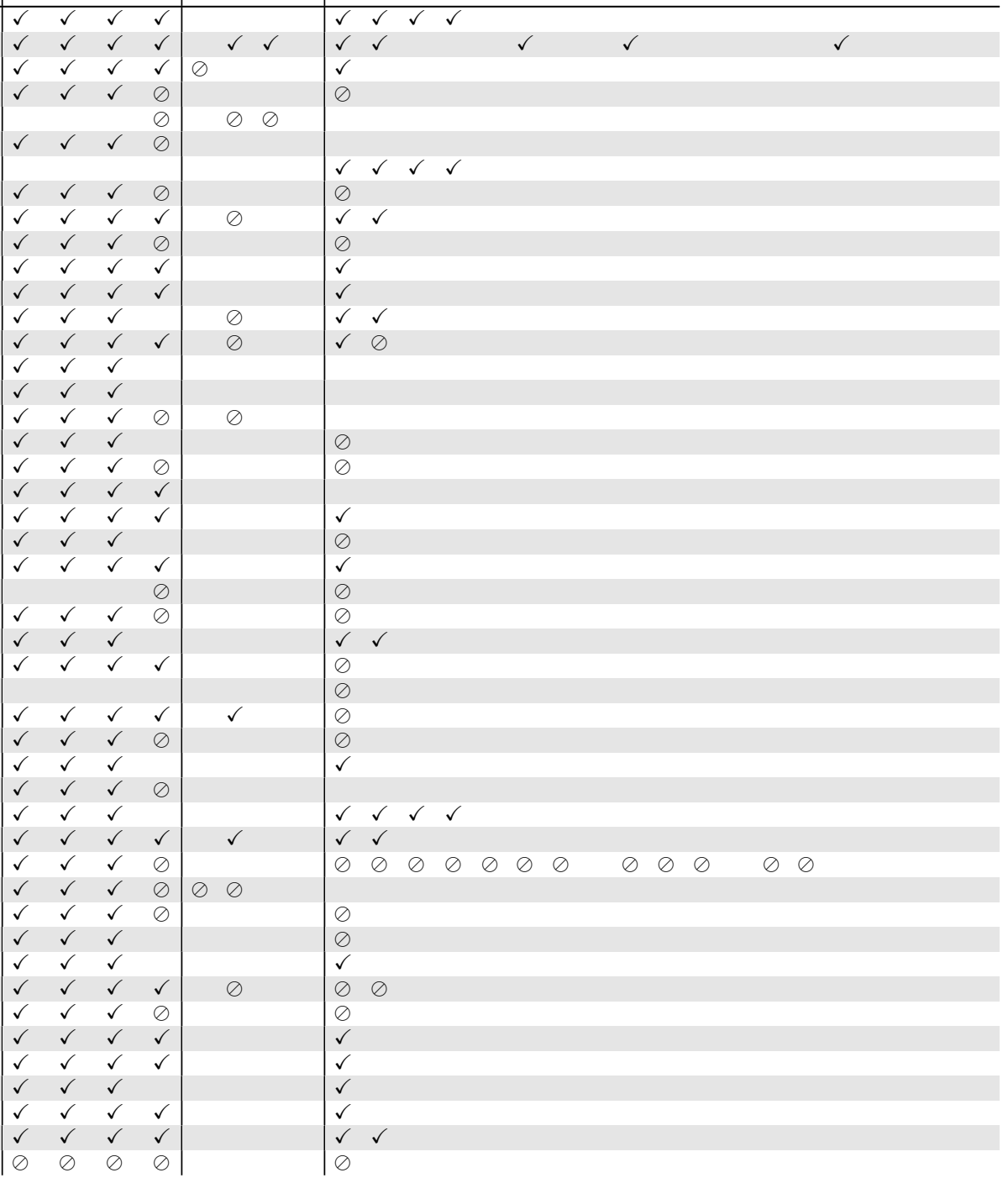
Within this work, the first comprehensive open pharmacokinetics data set on caffeine was established. We demonstrate the value of the data set by its application to multiple research questions relevant for metabolic phenotyping and liver function testing: (i) the effect of smoking and oral contraceptive use on caffeine elimination (Sec. 3.3); (ii) the interaction of caffeine with other drugs (Sec. 3.4), (iii) alteration of caffeine pharmacokinetics in disease (Sec. 3.5) and (iv) the applicability of caffeine as a salivary test substance by comparison of plasma and saliva data (Sec. 3.6). The data set integrates data from 148 publications (Fig. 1 and Tab. 1), with most of the publications corresponding to a distinct clinical trial. The focus of data curation was on pharmacokinetics data of caffeine, caffeine metabolites, and caffeine metabolic ratios in human adults. Importantly, the data set is enriched with meta-data on (i) the characteristics of studied patient cohorts and subjects (e.g. age, body weight, smoking status, health status, fasting); (ii) the applied interventions (e.g. dosing, substance, route of application); (iii) measured pharmacokinetic time-courses; and (iv) pharmacokinetic parameters (e.g. clearance, half-life, area under the curve). In summary, data from 1311 groups and 12106 individuals is reported under 1650 interventions resulting in 97026 pharmacokinetic outputs and 4224 time-courses. The data set is available via the pharmacokinetics database PK-DB (https://pk-db.com/) with a detailed description of the data structure provided in (Grzegorzewski et al. 2020). In the following, we summarize the quality of reporting and provide example applications of the data set.

\subsection{Reporting of pharmacokinetics data}

The study design as well as quality and details of reporting of results were very heterogeneous between studies. Major differences exist in the study design, number of study participants, and number of reported time-courses. Many studies report some individual participants data (84/148) but only a minority of the studies report individual participant data for all study participants (6/84) (Axelrod and Reichenthal, 1953; Brazier et al., 1980; McQuilkin et al., 1995; Millard et al., 2018; Perera et al., 2010; Rietveld et al., 1984). Many studies report only aggregated data on group level (64/148). In most studies, the application of a single dose of caffeine was studied (126/148). In the case of multiple interventions (55/148), mostly one additional substance was co-administrated (38/148). The main categories of studies were either (i) case-control studies which compare caffeine pharmacokinetics in two groups (e.g. healthy vs. disease) (62/148) , (ii) crossover studies on caffeine-drug interactions (comparing caffeine alone vs. caffeine and additional substance) (38/148) (iii) studies on metabolic phenotypes (including drug cocktails) (37/148); or (iv) methodological studies (e.g. establishing mass spectroscopy protocol for quantification or new site of sampling).

Intervention protocols, i.e. the applied substances, form, dose, and timing of application was typically reported in good detail. In crossover studies, the difference between treatments was generally reported in good detail. For the dosing with caffeine, the amount (140/148), route (e.g. oral, intravenous) (145/148), form (tablet, capsule, solution) (142/148), and the substance (148/148) are typically reported. Coadministrations of medication and other substances are often mentioned qualitatively (27/55), skipping either the amount, route, form, or exact timing of application.

The quantification protocol, i.e. quantification method (e.g. high-performance liquid chromatography, gas-liquid chromatography) (97/148), the site of sampling (e.g. plasma, serum, saliva, urine) (148/148), and the time points when samples were taken (129/148), were mostly reported in good detail. However, in some studies the quantification method and protocol are not mentioned explicitly but only via additional references, which complicates the curation.

The information on subject characteristics was less often reported in sufficient detail with large differences in the quality and quantity of reporting between studies. Any information on sex (137/148), 
weight (81/148), and age (94/148) was relatively often reported on group or individual level. However, age and weight were rarely reported on an individual level and often not even for all groups. Other anthropometric factors such as height (15/148), body mass index (BMI) (15/148), and ethnicity (26/148) are rarely reported. The genotype of CYP1A2 (gCYP1A2) is almost never reported (3/148), even though there is evidence that genetic variation can play an important role in caffeine metabolism. It is worth noting that low-cost genotyping methods were not available for early studies included in the data set. The phenotype (pCYP1A2, pXO, pNAT2) of enzymes involved in the metabolism of caffeine, i.e., CYP1A2, xanthine oxidase (XO), or N-acetyltransferase type 2 (NAT2) were investigated occasionally (37/148). The information on other factors influencing the pharmacokinetics of caffeine is reported very heterogeneously. The strong influence of smoking (106/148) and the use of oral contraceptives (42/148) on the enzyme activity of CYP1A2 and thereby on the apparent clearance of caffeine is covered relatively well in many publications. Health status and patient diseases are often covered (140/148). However, often categorized in broad and general disease classes, with more specific disease classification lacking. Markers related to cardiovascular health (e.g. blood pressure, cholesterol level, bilirubin level) are basically not reported in the context of caffeine pharmacokinetics. In case of cirrhosis, further information of severity is reported sparsely. Important information on the abstinence of caffeine or methylxanthines and consumption of caffeine or other caffeine-containing beverages is often missing.

Individual-level reporting is essential for subsequent pharmacokinetic modeling, as biological mechanisms responsible for the pharmacokinetics strongly correlate with these factors and large interindividual variability exists in caffeine pharmacokinetics. Despite the importance of individual subject data, information on individuals is rarely provided.

Most studies report pharmacokinetics outputs on caffeine (127/148). Data on the main product paraxanthine (46/148) and secondary metabolites theobromine (20/148) and theophylline (19/148) are reported sometimes. Additional metabolites such as 137U, 17U, 13U, 37U, 1X, 1U, 3X, 3U, 7X, 7U, AFMU, AAMU, ADMU, A1U, and A3U are seldom reported, mostly as urinary measurements. In summary, we established the largest freely available pharmacokinetics data set of caffeine so far consisting of data from 148 publications and clinical trials. By using a highly standardized data curation pipeline (established as part of PK-DB (Grzegorzewski et al., 2020)) high quality and consistency of the data could be achieved. This enabled us to apply the data set to various research questions relevant for metabolic phenotyping and liver function tests despite the heterogeneity in reporting. In the following, we demonstrate the value of the data set via multiple example applications consisting of (i) stratification of caffeine pharmacokinetics due to smoking and oral contraceptive use (Sec. 3.3), (ii) the interaction of drugs with caffeine (drug-drug interaction; Sec. 3.4); (iii) alteration of caffeine pharmacokinetics in disease (drug-disease interaction, Sec. 3.5, and (iv) metabolic phenotyping and liver function testing (Sec. 3.6).

\subsection{Smoking and oral contraceptives}

In a first analysis, we were interested in the effect of smoking and oral contraceptive use on the pharmacokinetics of caffeine in healthy subjects (see Fig. 2). Both have repeatedly been reported as key exogenous factors affecting caffeine elimination. A main question was how reproducible the effect is and if by integrating data from multiple studies a more consistent picture of the effects can be gained. For the analysis the data set was stratified into smokers, oral contraceptive users and a control group (neither smoking nor using oral contraceptives). Smoking results in increased caffeine clearance (Fig. 2A,C) and decreased half-life of caffeine elimination (Fig. 2B,D) whereas oral contraceptive use has the opposite effect over a wide dose range of caffeine. An important result from our analysis is that a consistent and 

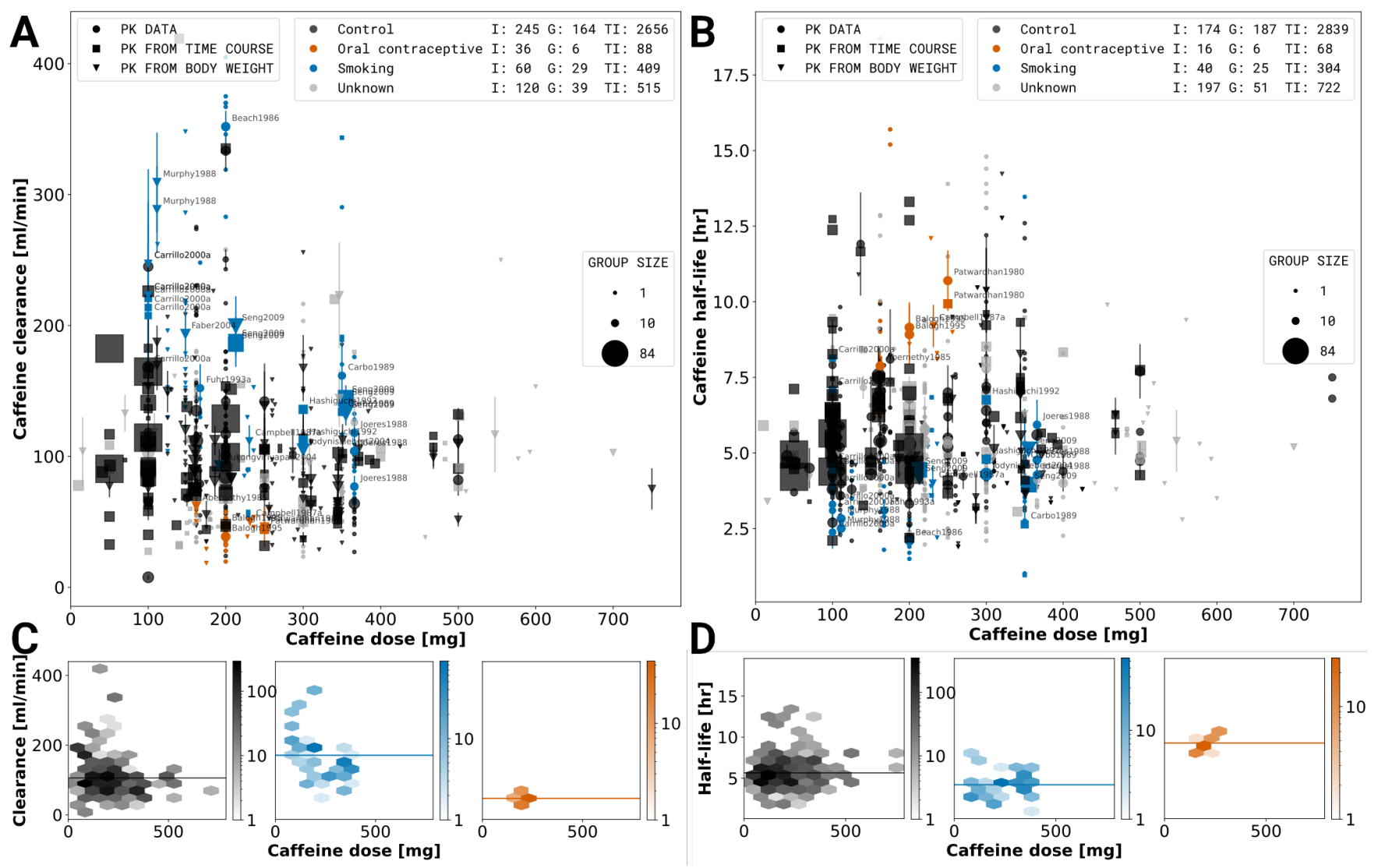

Figure 2. Dose-dependent effect of smoking and oral contraceptive use on caffeine pharmacokinetics. A stratified meta-analysis of caffeine clearance (A) and half-life (B) depending on reported smoking and oral contraceptive use was performed. Black: Control subjects are non-smokers and not taking oral contraceptives; Orange: Oral contraceptive users independent of smoking status (smokers and non-smokers). Blue: Smoking are smokers not consuming oral contraceptives. Grey: Unknown data correspond to subjects with unreported smoking and oral contraceptive status. Marker shape, and size describe the datatype and group size, respectively. Data representing smokers or oral contraceptive consumers is labeled by the respective study name. The hexagonal bin plots in the lower panel (C,D) correspond to the subset of data for the control, smoking, and oral contraceptive consuming subjects. The color intensity of each bin represents the number of subjects falling in a given hexagonal bin area. Data selection criteria and visualization are described in the methods section.

reproducible effect can be found over more than 50 years of pharmacokinetic research. With exception of a few outlier studies probably from a single clinical trial (see methods) all data was highly consistent. This provides a strong argument for the applied methods and protocols.

Despite the large effect of smoking and oral contraceptive use on the pharmacokinetics of caffeine, the information is only reported for a subset of studies. Smoking as well as oral contraceptive use should be an exclusion criteria for subjects in studies of caffeine pharmacokinetics, due to the possible confounding effects. Importantly, in many groups smokers and non-smokers were mixed without reporting data for smokers and non-smokers separately. Without reporting of data on individuals or subgroups no stratification could be performed, which could strongly affect results if not balanced between groups. In summary, the integrative data analysis showed a consistent strong activating effect of smoking on caffeine elimination and an inhibiting effect of oral contraceptive use on caffeine elimination. 

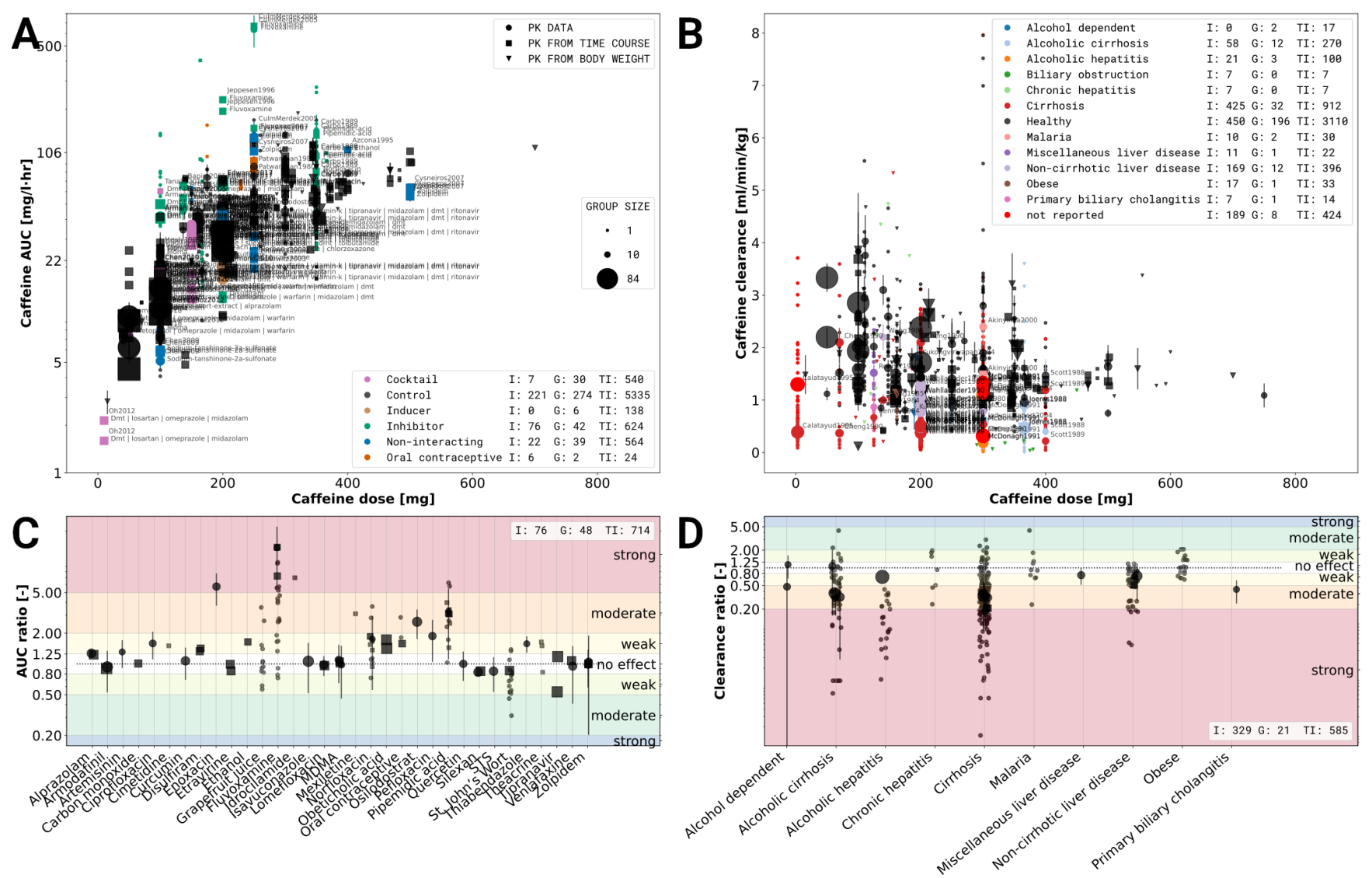

Figure 3. Effects of caffeine-drug and caffeine-disease interactions. A) Caffeine-drug interactions based on the caffeine area under the concentration curve (AUC). Data is stratified based on coadministration of drugs with caffeine. Violet: caffeine administrated as part of a drug cocktail. Common co-administrations are dextrometorphan, metoprolol, midazolam, omeprazole, and warfarin.; Black: single caffeine administration (no co-administration); Brown: co-administration with an inducing effect on the elimination of caffeine; Green: co-administration with an inhibiting effect on the elimination of caffeine; Blue: co-administrations with no effect on the pharmacokinetics of caffeine; Orange coadministration of oral contraceptives. B) Caffeine-disease interactions based on caffeine clearance. Data was stratified based on the health status and reported diseases, with black data points corresponding to healthy subjects. C) Effect sizes of caffeine-drug interaction for studies with a controlled study design, mostly randomized control trials (RCT). The effect size is based on the log AUC ratio between caffeine application alone and caffeine with co-administration of the respective drug. The drugs were characterized as having either a strong, moderate, weak or no effect. Strong, moderate and weak inhibitors increase the AUC $\geq 5$-fold, $\geq 2$ to $<5$-fold, $\geq 1.25$ to $<2$-fold, respectively. D) Effect size of caffeine-disease interactions for studies with a controlled study design, mostly case-controlled studies. The effect size is based on the log clearance ratio between subjects with and without a specific condition/disease. The diseases were characterized as having either a strong, moderate, weak or no effect. Strong, moderate and weak effect decreased the clearance by $\geq 80$ percent, $\geq 50$ to $<80$ percent and $\geq 20$ to $<50$ percent, respectively. Data selection criteria and visualization are described in the methods section.

\subsection{Caffeine-drug interactions}

An important question for metabolic phenotyping and liver function testing with caffeine is how the coadministration of other drugs and compounds affects caffeine clearance. Consequently, we studied in the second analysis the reported caffeine-drug interactions in the data set. The impact of drugs was quantified using the change in AUC between a coadministration and a respective control $\log \left(\frac{A U C_{\text {coadministration }}}{A U C_{\text {control }}}\right)$ (see Fig. 3A,C). 
Only case-controlled studies, mostly cross-over trials with a washout phase were included in the analysis. Corresponding controls were not matched across different studies. Overall coadministration data with AUC difference was available for 33 substances in our data set. In accordance with FDA, EMA and PMDA guidelines (Sudsakorn et al., 2020) we classified substances as inhibitors or inducers of caffeine clearance based on changes in AUC: FDA - Clinical DDI guidance: strong, moderate and weak inhibitors increase the AUC $\geq 5$-fold, $\geq 2$ to $<5$-fold, $\geq 1.25$ to $<2$-fold, respectively; strong, moderate and weak inducers decrease the AUC by $\geq 80$ percent, $\geq 50$ to $<80$ percent and $\geq 20$ to $<50$ percent, respectively.

Most substances do not affect the AUC of caffeine, with the exception of fluvoxamine, pipemidic acid and norfloxacin, which inhibit caffeine clearance. Tipranavir was the only substance showing a weak induction of caffeine clearance, but only in steady state dosing (not after a single dose) (Dumond et al. 2010)). Substances administered as a cocktail along side caffeine, aiming to phenotype several enzymes simultaneously did not affect the AUC of caffeine, substantiating the use of caffeine as part of a drug cocktail design (Armani et al., 2017; Dumond et al., 2010; Doroshyenko et al., 2013; Edwards et al., 2017; Kakuda et al., 2014; Lammers et al., 2018; Oh et al., 2012; Tanaka et al., 2014; Turpault et al., 2009). Our analysis shows that only a minority of studied drugs show an interaction with caffeine, confirming its value for phenotyping even under co-administration. As a side note, the protocols for studying caffeinedrug interactions were highly variable, e.g., the applied caffeine dose and the dose of the coadministrated substance varied between studies. Our results suggest that most medications can be safely consumed in combination with caffeine with exception of the antidepressant fluvoxamine, the antibacterial pipemidic acid and the antibiotic norfloxacin in which case caution is warranted.

\subsection{Caffeine-disease interactions}

An important question for using caffeine as a test substance for liver function testing and phenotyping, as well as for drugs metabolized via CYP1A2, is how disease affects the pharmacokinetics and elimination of caffeine. To study this question, we stratified caffeine clearance rates based on the reported disease of subjects and groups in the data set. To quantify the effect of disease the absolute clearance of caffeine (Fig. $3 \mathrm{~B}$ ) and the logarithmic difference to a control group $\log \left(\frac{A U C_{\text {disease }}}{A U C_{\text {control }}}\right)$ (Fig. $3 \mathrm{D}$ ) were analyzed. The corresponding controls were not matched across different studies.

None of the reported diseases increased the clearance rate of caffeine. Most of the diseases contained in this data set are diseases of the liver (e.g. alcoholic cirrhosis, primary biliary cholangitis) or are known to affect the liver (e.g. alcohol dependent). Cirrhotic liver disease had moderate to strong effects on the caffeine clearance with large variability in the reported data. Malaria and obesity had no effect on clearance with caffeine. An issue in the study of caffeine-disease interaction is that control group and disease group are different subjects (no cross-over design). In addition diseases were reported very heterogeneously (e.g. either only cirrhosis or with underlying cause such as alcoholic cirrhosis).

\subsection{Metabolic phenotyping}

An important question for metabolic phenotyping and liver function tests with caffeine is how saliva measurements correlate with plasma or serum caffeine measurements, in the following referred to as blood-based measurements. A good correlation would allow simple non-invasive phenotyping using saliva samples. To study this question we analyzed (i) the relationship of blood-based concentrations of caffeine and paraxanthine with their respective saliva concentrations (Fig. 4A,B); and (ii) how the caffeine clearance measured in saliva correlate to blood-based measurements (Fig. 4C).

Systematic errors due to different dosing protocols and different clinical investigation seem to be minimal as the data from multiple studies shows very consistent results. Linear regressions were performed to quantify the relation between saliva and blood-based caffeine and paraxanthine 

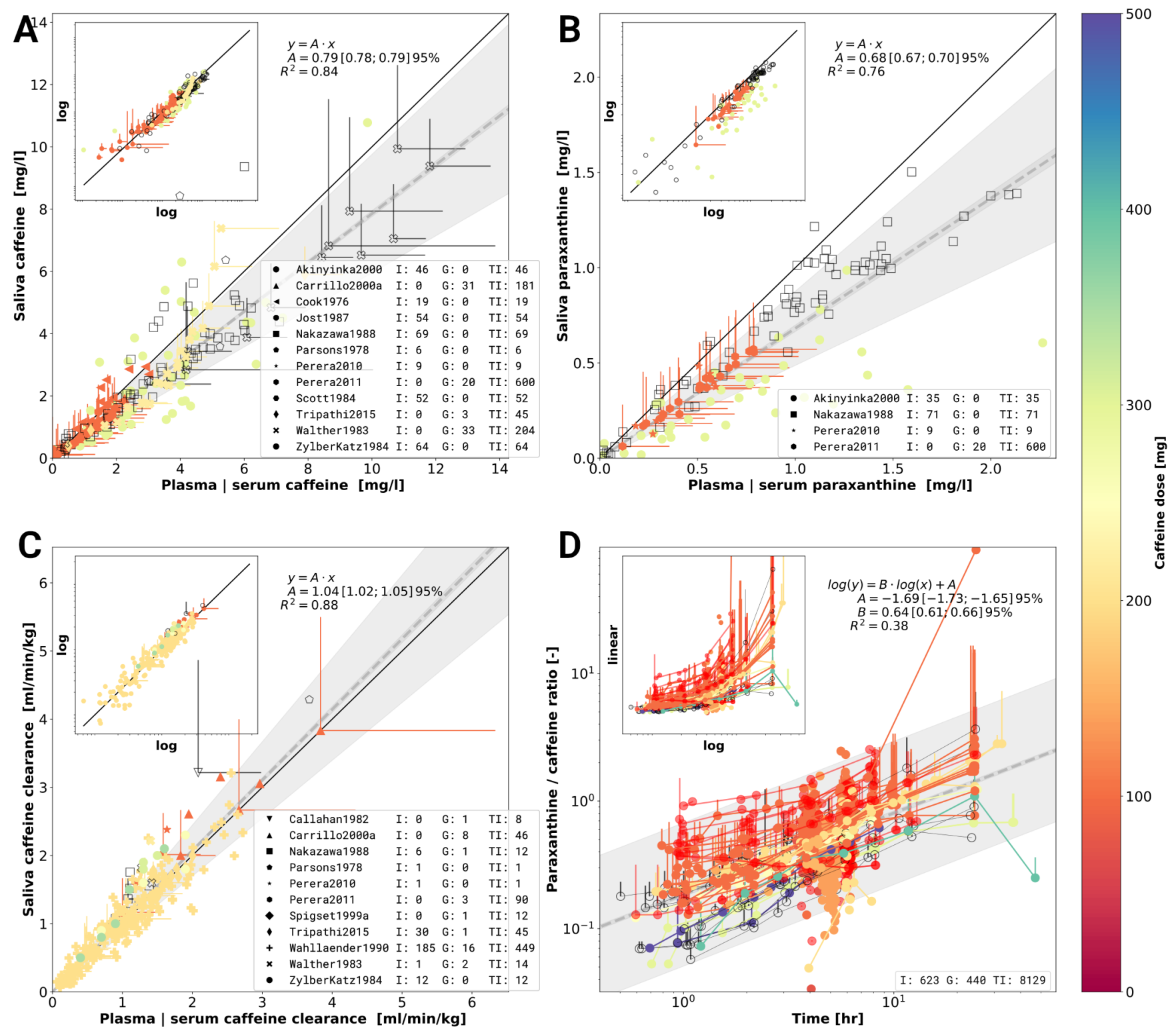

Figure 4. Meta-analysis of caffeine and paraxanthine concentrations in plasma, serum and saliva. A) Caffeine concentrations in saliva versus caffeine concentrations in plasma or serum. Individual data points come from a single investigation taken at identical times after caffeine dosing. Marker shape encodes the different study. Markers are color-coded by caffeine dose in mg. Empty markers correspond to data in which dosage was reported per body weight but no information on the subject weight was available. B) Paraxanthine concentrations in saliva versus paraxanthine concentrations in plasma or serum analogue to A). C) Caffeine clearance calculated from caffeine concentrations in saliva versus plasma or serum clearance. The panels A, B and C are in linear scale with a log-log inlet showing the same data. The dashed line in $\mathrm{A}, \mathrm{B}$ and $\mathrm{C}$ represents a linear regression $(y=A \cdot x)$ with wide shaded area being $95 \%$ confidence interval of the sample variability and narrow shaded area the $95 \%$ confidence interval of the fitted mean of the scaling factor $A$. D) Time dependency of the metabolic ratio paraxanthine/caffeine. Metabolic ratios are measured in plasma, serum, or saliva. Data points belonging to a single time course from a study are connected via a line. The dashed line corresponds to the linear regression $\log (y)=B \cdot \log (t)+A$. Jitter was applied on the time axes for better visibility of overlapping points. Data selection criteria and visualization are described in the methods section.

measurements (see Fig. 4A-C). The resulting scaling factors of saliva to blood-based concentration of caffeine and paraxanthine are $0.79 \pm 0.01$ and $0.68 \pm 0.02(\bar{x} \pm S D)$, respectively. Pearson correlation 
coefficients between saliva and blood-based concentrations for caffeine and paraxanthine are 0.84 and 0.76 , respectively.

When comparing saliva-based caffeine clearance against blood-based clearance (Fig. 4C) an even stronger correlation of 0.88 with a scaling factor of $01.04 \pm 0.02(\bar{x} \pm S D)$ is observed. The integrated clearance data strongly indicates that clearance can either be calculated from saliva or blood-based measurements.

Paraxanthine/caffeine ratios are mainly used for metabolic phenotyping based on caffeine. Whereas most studies use 6 hours to phenotype no clear consensus exists in the literature and metabolic ratios are reported for varying time points after caffeine application. Paraxanthine/caffeine ratios for caffeine administered either as a single dose or in a cocktail to healthy, non-smoking, non-pregnant, and non-oral contraceptive consuming subjects were investigated (Fig. 4D). By applying this strict data filtering, the variability due to either smoking and oral contraceptive use (see Sec. 3.3), caffeine-drug interactions (see Sec. 3.4) or disease (see Sec. 3.5) could be removed from the metabolic ratios.

Early and late time-sampling are least suitable for phenotyping. At these time points concentrations are low, resulting in relatively high random errors and thus low single to noise ratio. In an early stage, the outcome of metabolic ratios are further influenced by the distribution phase of the substance and its absorption kinetics, both affected by form and route of administration. Main results are that the metabolic phenotyping with paraxanthine/caffeine ratios is strongly time dependent with increasing ratios with time; and that a clear caffeine-dose dependency exists in the phenotyping with smaller caffeine doses increasing the metabolic ratio. Our results show the importance of clear standardized protocols for metabolic phenotyping.

In summary, by integrating data from multiple studies we could show a very good correlation between saliva and plasma caffeine concentrations, paraxanthine concentrations and pharmacokinetic parameters calculated from saliva versus plasma concentrations. This pooled data provides a strong argument for caffeine phenotyping based on saliva samples. Furthermore, we analyzed the time dependency of paraxanthine/caffeine ratios often used for phenotyping of CYP1A2. Our time dependent correlation allows to correct caffeine/paraxanthine ratios depending on the time after application and caffeine dose.

\section{DISCUSSION}

Within this work, we performed a systematic data integration and multiple data analyses of reported data on caffeine pharmacokinetics in adults with focus on applications in metabolic phenotyping and liver function testing. To our knowledge, this is the largest data set and analysis of its kind so far. Data integration from multiple sources allows to solidify existing knowledge, increases statistical power, increases generalizability, and creates new insights into the relationship between variables (Thacker. 1988). For instance, by systematically curating group and subject information on smoking status and oral contraceptive use, we could show a reproducible and consistent effect of smoking induction and inhibition via oral contraceptives over many studies and the complete dose regime of applied caffeine. Only a small subset of studies was specifically designed to study these questions (e.g. smoking by (Benowitz et al., 2003; Gunes et al., 2009; Parsons and Neims, 1978; Backman et al., 2008) and oral contraceptives by (Patwardhan et al., 1980; Abernethy and Todd, 1985; Rietveld et al., 1984)). Publicly providing comprehensive pharmacokinetics data in combination with detailed metadata allows to study new aspects of caffeine pharmacokinetics often not even anticipated by the original investigators. One example of such a new aspect is the determination of dose and time-dependency of metabolic phenotyping via paraxanthine/caffeine ratios besides many of the data sets only reporting data for a single time point 
and dose. Naturally, the advantages of open data are not exclusive to the study of caffeine, but apply to any given substance.

Like all data integration and meta-analysis methods limitations due to sampling bias, biased outcome interpretation and inadequate data may exist (Thacker, 1988). Despite being the most comprehensive analysis so far, we could only present selected aspects mainly driven by the availability of reported data. Our focus in this study was on key factors affecting caffeine pharmacokinetics (smoking and oral contraceptive use), caffeine-drug and caffeine-disease interactions, as well as information relevant for the metabolic phenotyping and liver function testing with caffeine. Other important factors such as the pharmacogenetics of caffeine or urinary metabolic ratios have not been presented. Importantly, the corresponding data has been curated and is readily available in PK-DB, but often very sparse (e.g. in case of genetic variants) or very heterogeneous (e.g. in case of urinary data). Despite many implemented measures to ensure high data quality (e.g. validation rules and checking of studies by multiple curators), we are aware that the created data set may contain mistakes. Please report such instances so that these can be resolved.

The scope of the presented data set is limited to caffeine pharmacokinetics in human adults. Future work will extend our data curation effort towards children and infants and include studies with animal experiment. In addition, despite trying to be as comprehensive as possible additional studies with relevant data exist. Please contact us in such cases so that we can include these additional studies. Contribution of missing data is highly appreciated. Also if you want to contribute a caffeine data set of your own please get in contact.

The large inter-individual variability in caffeine pharmacokinetics is a major limitation for metabolic phenotyping and liver function tests. Our work allowed to evaluate the effect of key factors on caffeine pharmacokinetics such as smoking, oral contraceptive usage or caffeine-drug interactions. An important next step will be the development of methods to quantify and correct for these confounding factors. This could allow to reduce variability in caffeine based testing. A promising tool in this context are physiological-based pharmacokinetics (PBPK) models (Jones and Rowland-Yeo, 2013) using information from the established data set as input for stratification and individualization. We could recently show that such an approach based on a similar data set for indocyanine green (ICG) allowed to account for important factors affecting ICG based liver function tests (Köller et al., 2021).

An important outcome of our analysis are the very good correlations between saliva- and blood-based measurements for caffeine with $0.77 R^{2}=0.74$ in very good agreement with data reported previously in individual studies $0.74 \pm 0.1 R^{2}=0.98$ (Akinyinka et al., 2000), $0.79 \pm 0.05 R^{2}=0.96$ (Zylber-Katz et al. 1984), $0.74 \pm 0.08$ (Newton et al., 1981), $0.74 \pm 0.08 R^{2}=0.90$ (Jost et al., 1987), $0.71 R^{2}=0.89$ (Scott et al. 1984), $0.73 \pm 0.06$ (Walther et al., 1983), and for paraxanthine $0.68 R^{2}=0.76$ compared to 0.77 $R^{2}=0.91$ (Nakazawa and Tanaka, 1988).

Several studies have shown that blood-derived pharmacokinetic parameters show excellent correlation with saliva-derived parameters (Akinyinka et al., 2000; Newton et al., 1981). We could confirm this observation when systematically analyzing the correlation between saliva- and plasma/serum-derived clearance. Overall we could show that the data from multiple studies are in very good agreement with each other after excluding data with confounding factors such as smoking or oral contraceptive use. These integrated results are a strong argument for saliva based metabolic phenotyping and liver function tests with caffeine, with sampling from saliva being convenient, painless, economical, without the requirement 
for special devices. Further, they allow simple repeated sampling as often required for pharmacokinetic research (Zylber-Katz et al., 1984).

Importantly, our results are not only applicable to caffeine, but many aspects can be translated to other substances metabolized via CYP1A2, e.g. to the LiMAx liver function tests based on the CYP1A2 substrate methacetin (Rubin et al., 2017). E.g. based on our analysis we would expect smoking and oral contraceptive use to be confounding factors of LiMAx tests, which should be recorded and be accounted for in the evaluation of liver function.

Our systematic curation and analysis of reported caffeine data provided an overview of the current state and limitations of reporting of pharmacokinetic data. In summary, an accepted standard, minimum information guidelines, and standardized meta-data for the reporting of pharmacokinetics data of caffeine are missing. This finding is apparently not only true for the pharmacokinetics of caffeine but rather generally true for reporting of pharmacokinetics research. Major shortcomings in reporting are missing minimum information on factors that are known to influence the pharmacokinetics of caffeine (e.g. smoking and oral contraceptive status). Often not even basic subject information (e.g. weight, sex, or age) are reported. These factors are essential in the analysis of the pharmacokinetics of any substance in vivo (Stader et al., 2019). In general-purpose pharmacokinetic data sets, concentration-time profiles are the fundamental and most valuable data type. Common practice however is not to report the raw measured data but only derived pharmacokinetic parameters or metabolic ratios. We strongly advocate the reporting of all data on an individual level while including detailed anonymized meta information alongside the concentration-time profiles. Access to the individual raw data would enable data integration with different data sets and the stratification of the data under various aspects. There are recent efforts in creating a standard resource for that matter (Grzegorzewski et al., 2020).

\section{CONFLICT OF INTEREST STATEMENT}

The authors declare that the research was conducted in the absence of any commercial or financial relationships that could be construed as a potential conflict of interest.

\section{AUTHOR CONTRIBUTIONS}

JG and MK designed the study, implemented and performed the analysis, curated the majority of caffeine studies and wrote the initial draft of the manuscript. FB and AK curated a subset of caffeine studies. All authors discussed the results. All authors contributed to and revised the manuscript critically.

\section{FUNDING}

JG, FB, AK and JG were supported by the Federal Ministry of Education and Research (BMBF, Germany) within the research network Systems Medicine of the Liver (LiSyM, grant number 031L0054). MK, FB and AK were supported by the German Research Foundation (DFG) within the Research Unit Programme FOR 5151 "QuaLiPerF (Quantifying Liver Perfusion-Function Relationship in Complex Resection - A Systems Medicine Approach)" by grant number 436883643.

\section{DATA AVAILABILITY STATEMENT}

The data sets analyzed for this study can be found in https://pk-db.com/. 


\section{Permission to Reuse and Copyright}

Figures, tables, and images will be published under a Creative Commons CC-BY licence and permission must be obtained for use of copyrighted material from other sources (including republished/adapted/modified/partial figures and images from the internet). It is the responsibility of the authors to acquire the licenses, to follow any citation instructions requested by third-party rights holders, and cover any supplementary charges.

\section{ACKNOWLEDGMENTS}

We acknowledge support by the BMBF-funded de.NBI Cloud within the German Network for Bioinformatics Infrastructure (de.NBI) (031A537B, 031A533A, 031A538A, 031A533B, 031A535A, 031A537C, 031A534A, 031A532B) for the PK-DB infrastructure.

\section{REFERENCES}

Abernethy, D. R. and Todd, E. L. (1985). Impairment of caffeine clearance by chronic use of low-dose oestrogen-containing oral contraceptives. European journal of clinical pharmacology 28, 425-8

Abernethy, D. R., Todd, E. L., and Schwartz, J. B. (1985). Caffeine disposition in obesity. British journal of clinical pharmacology 20, 61-6

Akinyinka, O. O., Sowunmi, A., Honeywell, R., and Renwick, A. G. (2000). The effects of acute falciparum malaria on the disposition of caffeine and the comparison of saliva and plasma-derived pharmacokinetic parameters in adult nigerians. European journal of clinical pharmacology 56, 159-65

Amchin, J., Zarycranski, W., Taylor, K. P., Albano, D., and Klockowski, P. M. (1999). Effect of venlafaxine on cyp1a2-dependent pharmacokinetics and metabolism of caffeine. Journal of clinical pharmacology 39, 252-9

Armani, S., Ting, L., Sauter, N., Darstein, C., Tripathi, A. P., Wang, L., et al. (2017). Drug interaction potential of osilodrostat (lci699) based on its effect on the pharmacokinetics of probe drugs of cytochrome p450 enzymes in healthy adults. Clinical drug investigation 37, 465-472. doi:10.1007/s40261-017-0497-0

Arnaud, M. J. (2011). Pharmacokinetics and metabolism of natural methylxanthines in animal and man. Handbook of experimental pharmacology, 33-91doi:10.1007/978-3-642-13443-2_3

[Dataset] Arnaud, M. J. and Welsch, C. (1982). Theophylline and caffeine metabolism in man

Arold, G., Donath, F., Maurer, A., Diefenbach, K., Bauer, S., Henneicke-von Zepelin, H.-H., et al. (2005). No relevant interaction with alprazolam, caffeine, tolbutamide, and digoxin by treatment with a low-hyperforin st john's wort extract. Planta medica 71, 331-7

Axelrod, J. and Reichenthal, J. (1953). The fate of caffeine in man and a method for its estimation in biological material. The Journal of pharmacology and experimental therapeutics 107, 519-23

Azcona, O., Barbanoj, M. J., Torrent, J., and Jané, F. (1995). Evaluation of the central effects of alcohol and caffeine interaction. British journal of clinical pharmacology 40, 393-400. doi:10.1111/j. 1365-2125.1995.tb04562.x

Backman, J. T., Schröder, M. T., and Neuvonen, P. J. (2008). Effects of gender and moderate smoking on the pharmacokinetics and effects of the cyp1a2 substrate tizanidine. European journal of clinical pharmacology 64, 17-24

Balogh, A., Harder, S., Vollandt, R., and Staib, A. H. (1992). Intra-individual variability of caffeine elimination in healthy subjects. International journal of clinical pharmacology, therapy, and toxicology $30,383-7$ 
Balogh, A., Klinger, G., Henschel, L., Börner, A., Vollanth, R., and Kuhnz, W. (1995). Influence of ethinylestradiol-containing combination oral contraceptives with gestodene or levonorgestrel on caffeine elimination. European journal of clinical pharmacology 48, 161-6

Bapiro, T. E., Sayi, J., Hasler, J. A., Jande, M., Rimoy, G., Masselle, A., et al. (2005). Artemisinin and thiabendazole are potent inhibitors of cytochrome p450 1a2 (cyp1a2) activity in humans. European journal of clinical pharmacology 61, 755-61

Barnett, G., Segura, J., de la Torre, R., and Carbó, M. (1990). Pharmacokinetic determination of relative potency of quinolone inhibition of caffeine disposition. European journal of clinical pharmacology 39, 63-9

Bchir, F., Dogui, M., Ben Fradj, R., Arnaud, M. J., and Saguem, S. (2006). Differences in pharmacokinetic and electroencephalographic responses to caffeine in sleep-sensitive and non-sensitive subjects. Comptes rendus biologies 329, 512-9

Beach, C. A., Mays, D. C., Guiler, R. C., Jacober, C. H., and Gerber, N. (1986). Inhibition of elimination of caffeine by disulfiram in normal subjects and recovering alcoholics. Clinical pharmacology and therapeutics 39, 265-70

Becker, A. B., Simons, K. J., Gillespie, C. A., and Simons, F. E. (1984). The bronchodilator effects and pharmacokinetics of caffeine in asthma. The New England journal of medicine 310, 743-6

Begas, E., Kouvaras, E., Tsakalof, A., Papakosta, S., and Asprodini, E. K. (2007). In vivo evaluation of cyp1a2, cyp2a6, nat-2 and xanthine oxidase activities in a greek population sample by the rp-hplc monitoring of caffeine metabolic ratios. Biomedical chromatography : BMC 21, 190-200

Begas, E., Kouvaras, E., Tsakalof, A. K., Bounitsi, M., and Asprodini, E. K. (2015). Development and validation of a reversed-phase hplc method for cyp1a2 phenotyping by use of a caffeine metabolite ratio in saliva. Biomedical chromatography : BMC 29, 1657-63

Benowitz, N. L., Jacob, P. r., Mayan, H., and Denaro, C. (1995). Sympathomimetic effects of paraxanthine and caffeine in humans. Clinical pharmacology and therapeutics 58, 684-91

Benowitz, N. L., Peng, M., and Jacob, P. (2003). Effects of cigarette smoking and carbon monoxide on chlorzoxazone and caffeine metabolism. Clinical pharmacology and therapeutics 74, 468-474. doi:10.1016/j.clpt.2003.07.001

Birkett, D. J. and Miners, J. O. (1991). Caffeine renal clearance and urine caffeine concentrations during steady state dosing. implications for monitoring caffeine intake during sports events. British journal of clinical pharmacology 31, 405-8

Blanchard, J. and Sawers, S. J. (1983a). The absolute bioavailability of caffeine in man. European journal of clinical pharmacology 24, 93-8

Blanchard, J. and Sawers, S. J. (1983b). Comparative pharmacokinetics of caffeine in young and elderly men. Journal of pharmacokinetics and biopharmaceutics 11, 109-26

Blanchard, J. and Sawers, S. J. (1983c). Relationship between urine flow rate and renal clearance of caffeine in man. Journal of clinical pharmacology 23, 134-8

Blanchard, J., Sawers, S. J., Jonkman, J. H., and Tang-Liu, D. D. (1985). Comparison of the urinary metabolite profile of caffeine in young and elderly males. British journal of clinical pharmacology 19 , 225-232. doi:10.1111/j.1365-2125.1985.tb02635.x

Bonati, M., Latini, R., Galletti, F., Young, J. F., Tognoni, G., and Garattini, S. (1982). Caffeine disposition after oral doses. Clinical pharmacology and therapeutics 32, 98-106

Bozikas, V. P., Papakosta, M., Niopas, I., Karavatos, A., and Mirtsou-Fidani, V. (2004). Smoking impact on cyp1a2 activity in a group of patients with schizophrenia. European neuropsychopharmacology : the journal of the European College of Neuropsychopharmacology 14, 39-44 
Brazier, J. L., Descotes, J., Lery, N., Ollagnier, M., and Evreux, J. C. (1980). Inhibition by idrocilamide of the disposition of caffeine. European journal of clinical pharmacology 17, 37-43. doi:10.1007/ BF00561675

Broughton, L. J. and Rogers, H. J. (1981). Decreased systemic clearance of caffeine due to cimetidine. British journal of clinical pharmacology 12, 155-9

Bruce, M., Scott, N., Lader, M., and Marks, V. (1986). The psychopharmacological and electrophysiological effects of single doses of caffeine in healthy human subjects. British journal of clinical pharmacology 22, 81-7

Calatayud, O., Rodríguez, M., Sánchez-Alcaraz, A., and Ibáñez, P. (1995). Caffeine test assessment for measuring liver function in critically ill patients. Journal of clinical pharmacy and therapeutics 20, 23-9

Callahan, M. M., Robertson, R. S., Arnaud, M. J., Branfman, A. R., McComish, M. F., and Yesair, D. W. (1982). Human metabolism of [1-methyl-14c]- and [2-14c]caffeine after oral administration. Drug metabolism and disposition: the biological fate of chemicals 10, 417-423

Callahan, M. M., Robertson, R. S., Branfman, A. R., McComish, M. F., and Yesair, D. W. (1983). Comparison of caffeine metabolism in three nonsmoking populations after oral administration of radiolabeled caffeine. Drug metabolism and disposition: the biological fate of chemicals 11, 211-7

Campbell, M. E., Spielberg, S. P., and Kalow, W. (1987). A urinary metabolite ratio that reflects systemic caffeine clearance. Clinical pharmacology and therapeutics 42, 157-65

Carbó, M., Segura, J., De la Torre, R., Badenas, J. M., and Camí, J. (1989). Effect of quinolones on caffeine disposition. Clinical pharmacology and therapeutics 45, 234-40

Carrillo, J. A., Christensen, M., Ramos, S. I., Alm, C., Dahl, M. L., Benitez, J., et al. (2000). Evaluation of caffeine as an in vivo probe for cyp1a2 using measurements in plasma, saliva, and urine. Therapeutic drug monitoring 22, 409-17

Cattarossi, L., Colacino, V., Janes, A., LoGreco, P., Rubini, S., Zilli, M., et al. (1988). Oral versus intramuscular loading of caffeine in idiopathic apnoea of prematurity. European journal of pediatrics $148,262-3$

Chen, Y., Liu, W.-H., Chen, B.-L., Fan, L., Han, Y., Wang, G., et al. (2010). Plant polyphenol curcumin significantly affects cyp1a2 and cyp2a6 activity in healthy, male chinese volunteers. The Annals of pharmacotherapy 44, 1038-1045. doi:10.1345/aph.1M533

Chen, Y., Tu, J.-H., He, Y.-J., Zhang, W., Wang, G., Tan, Z.-R., et al. (2009a). Effect of sodium tanshinone ii a sulfonate on the activity of cyp1a2 in healthy volunteers. Xenobiotica; the fate of foreign compounds in biological systems 39, 508-513. doi:10.1080/00498250902951763

Chen, Y., Xiao, P., Ou-Yang, D.-S., Fan, L., Guo, D., Wang, Y.-N., et al. (2009b). Simultaneous action of the flavonoid quercetin on cytochrome p450 (cyp) 1a2, cyp2a6, n-acetyltransferase and xanthine oxidase activity in healthy volunteers. Clinical and experimental pharmacology \& physiology 36, 828-833. doi:10.1111/j.1440-1681.2009.05158.x

Cheng, W. S., Murphy, T. L., Smith, M. T., Cooksley, W. G., Halliday, J. W., and Powell, L. W. (1990). Dose-dependent pharmacokinetics of caffeine in humans: relevance as a test of quantitative liver function. Clinical pharmacology and therapeutics 47, 516-24

Chia, H. Y., Yau, W.-P., and Ho, H. K. (2016). Establishing population distribution of drug-metabolizing enzyme activities for the use of salivary caffeine as a dynamic liver function marker in a singaporean chinese population. Biopharmaceutics \& drug disposition 37, 168-81

Christensen, M., Tybring, G., Mihara, K., Yasui-Furokori, N., Carrillo, J. A., Ramos, S. I., et al. (2002). Low daily 10-mg and 20-mg doses of fluvoxamine inhibit the metabolism of both caffeine (cytochrome 
p4501a2) and omeprazole (cytochrome p4502c19). Clinical pharmacology and therapeutics 71, 14152

Clements, J. A., Critchley, J. A., and Prescott, L. F. (1984). The role of sulphate conjugation in the metabolism and disposition of oral and intravenous paracetamol in man. British journal of clinical pharmacology $18,481-5$

Cook, C. E., Tallent, C. R., Amerson, E. W., Myers, M. W., Kepler, J. A., Taylor, G. F., et al. (1976). Caffeine in plasma and saliva by a radioimmunoassay procedure. The Journal of pharmacology and experimental therapeutics 199, 679-686

Cornish, H. H. and Christman, A. A. (1957). A study of the metabolism of theobromine, theophylline, and caffeine in man. The Journal of biological chemistry 228, 315-23

Culm-Merdek, K. E., von Moltke, L. L., Harmatz, J. S., and Greenblatt, D. J. (2005). Fluvoxamine impairs single-dose caffeine clearance without altering caffeine pharmacodynamics. British journal of clinical pharmacology 60, 486-93

Cysneiros, R. M., Farkas, D., Harmatz, J. S., von Moltke, L. L., and Greenblatt, D. J. (2007). Pharmacokinetic and pharmacodynamic interactions between zolpidem and caffeine. Clinical pharmacology and therapeutics $82,54-62$

Darwish, M., Kirby, M., Robertson, P. J., and Hellriegel, E. T. (2008). Interaction profile of armodafinil with medications metabolized by cytochrome p450 enzymes 1a2, 3a4 and 2c19 in healthy subjects. Clinical pharmacokinetics 47, 61-74

Desmond, P. V., Patwardhan, R. V., Johnson, R. F., and Schenker, S. (1980). Impaired elimination of caffeine in cirrhosis. Digestive diseases and sciences 25, 193-7

Dinger, J., Woods, C., Brandt, S. D., Meyer, M. R., and Maurer, H. H. (2016). Cytochrome p450 inhibition potential of new psychoactive substances of the tryptamine class. Toxicology letters 241 , 82-94. doi:10.1016/j.toxlet.2015.11.013

Djordjevic, N., Ghotbi, R., Bertilsson, L., Jankovic, S., and Aklillu, E. (2008). Induction of cyp1a2 by heavy coffee consumption in serbs and swedes. European journal of clinical pharmacology 64, 381-5

Doroshyenko, O., Rokitta, D., Zadoyan, G., Klement, S., Schläfke, S., Dienel, A., et al. (2013). Drug cocktail interaction study on the effect of the orally administered lavender oil preparation silexan on cytochrome p450 enzymes in healthy volunteers. Drug metabolism and disposition: the biological fate of chemicals 41, 987-93

Drozdzik, M., Busch, D., Lapczuk, J., Müller, J., Ostrowski, M., Kurzawski, M., et al. (2018). Protein abundance of clinically relevant drug-metabolizing enzymes in the human liver and intestine: A comparative analysis in paired tissue specimens. Clinical pharmacology and therapeutics 104, 515-524. doi:10.1002/cpt.967

Dumond, J. B., Vourvahis, M., Rezk, N. L., Patterson, K. B., Tien, H.-C., White, N., et al. (2010). A phenotype-genotype approach to predicting cyp450 and p-glycoprotein drug interactions with the mixed inhibitor/inducer tipranavir/ritonavir. Clinical pharmacology and therapeutics 87, 735-742. doi:10.1038/clpt.2009.253

Edwards, J. E., Eliot, L., Parkinson, A., Karan, S., and MacConell, L. (2017). Assessment of pharmacokinetic interactions between obeticholic acid and caffeine, midazolam, warfarin, dextromethorphan, omeprazole, rosuvastatin, and digoxin in phase 1 studies in healthy subjects. Advances in therapy 34, 2120-2138. doi:10.1007/s12325-017-0601-0

Faber, M. S. and Fuhr, U. (2004). Time response of cytochrome p450 1a2 activity on cessation of heavy smoking. Clinical pharmacology and therapeutics 76, 178-84 
Faber, M. S., Jetter, A., and Fuhr, U. (2005). Assessment of cyp1a2 activity in clinical practice: why, how, and when? Basic \& clinical pharmacology \& toxicology 97, 125-134. doi:10.1111/j.1742-7843.2005. pto_973160.x

Frary, C. D., Johnson, R. K., and Wang, M. Q. (2005). Food sources and intakes of caffeine in the diets of persons in the united states. Journal of the American Dietetic Association 105, 110-113. doi:10.1016/j.jada.2004.10.027

Fuhr, U., Klittich, K., and Staib, A. H. (1993). Inhibitory effect of grapefruit juice and its bitter principal, naringenin, on cyp1a2 dependent metabolism of caffeine in man. British journal of clinical pharmacology 35, 431-6

Fuhr, U., Rost, K. L., Engelhardt, R., Sachs, M., Liermann, D., Belloc, C., et al. (1996). Evaluation of caffeine as a test drug for cyp1a2, nat2 and cyp2e1 phenotyping in man by in vivo versus in vitro correlations. Pharmacogenetics 6, 159-176. doi:10.1097/00008571-199604000-00003

Furge, L. L. and Fletke, K. J. (2007). Hplc determination of caffeine and paraxanthine in urine: An assay for cytochrome p450 1a2 activity. Biochemistry and molecular biology education : a bimonthly publication of the International Union of Biochemistry and Molecular Biology 35, 138-44

George, J., Murphy, T., Roberts, R., Cooksley, W. G., Halliday, J. W., and Powell, L. W. (1986). Influence of alcohol and caffeine consumption on caffeine elimination. Clinical and experimental pharmacology \& physiology $13,731-6$

Ghassabian, S., Chetty, M., Tattam, B. N., Chem, M. C., Glen, J., Rahme, J., et al. (2009). A highthroughput assay using liquid chromatography-tandem mass spectrometry for simultaneous in vivo phenotyping of 5 major cytochrome p450 enzymes in patients. Therapeutic drug monitoring 31, 23946

Ghotbi, R., Christensen, M., Roh, H.-K., Ingelman-Sundberg, M., Aklillu, E., and Bertilsson, L. (2007). Comparisons of cyp1a2 genetic polymorphisms, enzyme activity and the genotype-phenotype relationship in swedes and koreans. European journal of clinical pharmacology 63, 537-546. doi:10.1007/s00228-007-0288-2

Gilbert, R. (1984). The methylxanthine beverages and foods: chemistry, consumption, and health effects. Progress in clinical and biological research 158, 1-413

Granfors, M. T., Backman, J. T., Laitila, J., and Neuvonen, P. J. (2005). Oral contraceptives containing ethinyl estradiol and gestodene markedly increase plasma concentrations and effects of tizanidine by inhibiting cytochrome p450 1a2. Clinical pharmacology and therapeutics 78, 400-11

Grzegorzewski, J., Brandhorst, J., Green, K., Eleftheriadou, D., Duport, Y., Barthorscht, F., et al. (2020). Pk-db: pharmacokinetics database for individualized and stratified computational modeling. Nucleic acids research doi:10.1093/nar/gkaa990

Gunes, A. and Dahl, M.-L. (2008). Variation in cyp1a2 activity and its clinical implications: influence of environmental factors and genetic polymorphisms. Pharmacogenomics 9, 625-637. doi:10.2217/ 14622416.9.5.625

Gunes, A., Ozbey, G., Vural, E. H., Uluoglu, C., Scordo, M. G., Zengil, H., et al. (2009). Influence of genetic polymorphisms, smoking, gender and age on cyp1a2 activity in a turkish population. Pharmacogenomics 10, 769-778. doi:10.2217/pgs.09.22

Gurley, B. J., Gardner, S. F., Hubbard, M. A., Williams, D. K., Gentry, W. B., Cui, Y., et al. (2005a). Clinical assessment of effects of botanical supplementation on cytochrome p450 phenotypes in the elderly: St john's wort, garlic oil, panax ginseng and ginkgo biloba. Drugs \& aging 22, 525-539. doi:10.2165/00002512-200522060-00006 
Gurley, B. J., Gardner, S. F., Hubbard, M. A., Williams, D. K., Gentry, W. B., Khan, I. A., et al. (2005b). In vivo effects of goldenseal, kava kava, black cohosh, and valerian on human cytochrome p450 1a2, 2d6, 2e1, and 3a4/5 phenotypes. Clinical pharmacology and therapeutics 77, 415-426. doi:10.1016/j. clpt.2005.01.009

Hakooz, N. M. K. (2009). Caffeine metabolic ratios for the in vivo evaluation of cyp1a2, nacetyltransferase 2, xanthine oxidase and cyp2a6 enzymatic activities. Current drug metabolism 10, 329-338. doi:10.2174/138920009788499003

Haller, C. A., Jacob, P. r., and Benowitz, N. L. (2002). Pharmacology of ephedra alkaloids and caffeine after single-dose dietary supplement use. Clinical pharmacology and therapeutics 71, 421-32

Hamon-Vilcot, B., Simon, T., Becquemont, L., Poirier, J.-M., Piette, F., and Jaillon, P. (2004). Effects of malnutrition on cytochrome p450 1a2 activity in elderly patients. Therapie 59, 247-251. doi:10.2515/ therapie:2004048

Harder, S., Fuhr, U., Staib, A. H., and Wolff, T. (1989). Ciprofloxacin-caffeine: a drug interaction established using in vivo and in vitro investigations. The American journal of medicine 87, 89S-91S

Harder, S., Staib, A. H., Beer, C., Papenburg, A., Stille, W., and Shah, P. M. (1988). 4-quinolones inhibit biotransformation of caffeine. European journal of clinical pharmacology 35, 651-6

Hashiguchi, M., Fujimura, A., Ohashi, K., and Ebihara, A. (1992). Diurnal effect on caffeine clearance. Journal of clinical pharmacology 32, 184-187. doi:10.1002/j.1552-4604.1992.tb03824.x

He, H., Ma, D., Crone, L. B., Butawan, M., Meibohm, B., Bloomer, R. J., et al. (2017). Assessment of the drug-drug interaction potential between theacrine and caffeine in humans. Journal of caffeine research 7, 95-102

Healy, D. P., Polk, R. E., Kanawati, L., Rock, D. T., and Mooney, M. L. (1989). Interaction between oral ciprofloxacin and caffeine in normal volunteers. Antimicrobial agents and chemotherapy 33, 474-8

Healy, D. P., Schoenle, J. R., Stotka, J., and Polk, R. E. (1991). Lack of interaction between lomefloxacin and caffeine in normal volunteers. Antimicrobial agents and chemotherapy 35, 660-4

Hetzler, R. K., Knowlton, R. G., Somani, S. M., Brown, D. D., and Perkins, R. M. r. (1990). Effect of paraxanthine on ffa mobilization after intravenous caffeine administration in humans. Journal of applied physiology (Bethesda, Md. : 1985) 68, 44-7

Holstege, A., Kurz, M., Weinbeck, M., and Gerok, W. (1993). Excretion of caffeine and its primary degradation products into bile. Journal of hepatology 17, 67-73

Holstege, A., Staiger, M., Haag, K., and Gerok, W. (1989). Correlation of caffeine elimination and child's classification in liver cirrhosis. Klinische Wochenschrift 67, 6-15

Iqbal, N., Ahmad, B., Janbaz, K. H., Gilani, A. U., and Niazi, S. K. (1995). The effect of caffeine on the pharmacokinetics of acetaminophen in man. Biopharmaceutics \& drug disposition 16, 481-7

Jeppesen, U., Loft, S., Poulsen, H. E., and Brśen, K. (1996). A fluvoxamine-caffeine interaction study. Pharmacogenetics 6, 213-22

Jiang, Z., Dragin, N., Jorge-Nebert, L. F., Martin, M. V., Guengerich, F. P., Aklillu, E., et al. (2006). Search for an association between the human cyp1a2 genotype and cyp1a2 metabolic phenotype. Pharmacogenetics and genomics 16, 359-367. doi:10.1097/01.fpc.0000204994.99429.46

Jodynis-Liebert, J., Flieger, J., Matuszewska, A., and Juszczyk, J. (2004). Serum metabolite/caffeine ratios as a test for liver function. Journal of clinical pharmacology 44, 338-47

Joeres, R., Klinker, H., Heusler, H., Epping, J., and Richter, E. (1987). Influence of mexiletine on caffeine elimination. Pharmacology \& therapeutics 33, 163-169. doi:10.1016/0163-7258(87)90046-5

Joeres, R., Klinker, H., Heusler, H., Epping, J., Zilly, W., and Richter, E. (1988). Influence of smoking on caffeine elimination in healthy volunteers and in patients with alcoholic liver cirrhosis. Hepatology 
(Baltimore, Md.) 8, 575-9

Jones, H. and Rowland-Yeo, K. (2013). Basic concepts in physiologically based pharmacokinetic modeling in drug discovery and development. CPT: pharmacometrics \& systems pharmacology 2 , e63. doi:10.1038/psp.2013.41

Jost, G., Wahlländer, A., von Mandach, U., and Preisig, R. (1987). Overnight salivary caffeine clearance: a liver function test suitable for routine use. Hepatology (Baltimore, Md.) 7, 338-344. doi:10.1002/ hep. 1840070221

Kakuda, T. N., Van Solingen-Ristea, R. M., Onkelinx, J., Stevens, T., Aharchi, F., De Smedt, G., et al. (2014). The effect of single- and multiple-dose etravirine on a drug cocktail of representative cytochrome p450 probes and digoxin in healthy subjects. Journal of clinical pharmacology 54, 422-31

Kalow, W. and Tang, B. K. (1993). The use of caffeine for enzyme assays: a critical appraisal. Clinical pharmacology and therapeutics 53, 503-514. doi:10.1038/clpt.1993.63

Kamimori, G. H., Joubert, A., Otterstetter, R., Santaromana, M., and Eddington, N. D. (1999). The effect of the menstrual cycle on the pharmacokinetics of caffeine in normal, healthy eumenorrheic females. European journal of clinical pharmacology 55, 445-9

Kamimori, G. H., Karyekar, C. S., Otterstetter, R., Cox, D. S., Balkin, T. J., Belenky, G. L., et al. (2002). The rate of absorption and relative bioavailability of caffeine administered in chewing gum versus capsules to normal healthy volunteers. International journal of pharmaceutics 234, 159-67

Kamimori, G. H., Somani, S. M., Knowlton, R. G., and Perkins, R. M. (1987). The effects of obesity and exercise on the pharmacokinetics of caffeine in lean and obese volunteers. European journal of clinical pharmacology 31, 595-600. doi:10.1007/BF00606637

Kaplan, G. B., Greenblatt, D. J., Ehrenberg, B. L., Goddard, J. E., Cotreau, M. M., Harmatz, J. S., et al. (1997). Dose-dependent pharmacokinetics and psychomotor effects of caffeine in humans. Journal of clinical pharmacology 37, 693-703

Kinzig-Schippers, M., Fuhr, U., Zaigler, M., Dammeyer, J., Rüsing, G., Labedzki, A., et al. (1999). Interaction of pefloxacin and enoxacin with the human cytochrome p450 enzyme cyp1a2. Clinical pharmacology and therapeutics 65, 262-274. doi:10.1016/S0009-9236(99)70105-0

Klein, K., Winter, S., Turpeinen, M., Schwab, M., and Zanger, U. M. (2010). Pathway-targeted pharmacogenomics of cyp1a2 in human liver. Frontiers in pharmacology 1, 129. doi:10.3389/fphar. 2010.00129

Koch, J. P., ten Tusscher, G. W., Koppe, J. G., and Guchelaar, H. J. (1999). Validation of a highperformance liquid chromatography assay for quantification of caffeine and paraxanthine in human serum in the context of cyp1a2 phenotyping. Biomedical chromatography : BMC 13, 309-14

Köller, A., Grzegorzewski, J., Tautenhahn, H.-M., and König, M. (2021). Prediction of survival after partial hepatectomy using a physiologically based pharmacokinetic model of indocyanine green liver function tests. bioRxiv doi:10.1101/2021.06.15.448411

Kot, M. and Daniel, W. A. (2008). Caffeine as a marker substrate for testing cytochrome p450 activity in human and rat. Pharmacological reports : PR 60, 789-797

Kukongviriyapan, V., Senggunprai, L., Prawan, A., Gaysornsiri, D., Kukongviriyapan, U., and AiemsaArd, J. (2004). Salivary caffeine metabolic ratio in alcohol-dependent subjects. European journal of clinical pharmacology 60, 103-7

Laizure, S. C., Meibohm, B., Nelson, K., Chen, F., Hu, Z.-Y., and Parker, R. B. (2017). Comparison of caffeine disposition following administration by oral solution (energy drink) and inspired powder (aeroshot) in human subjects. British journal of clinical pharmacology 83, 2687-2694 
Lammers, L. A., Achterbergh, R., Romijn, J. A., and Mathôt, R. A. A. (2018). Short-term fasting alters pharmacokinetics of cytochrome p450 probe drugs: Does protein binding play a role? European journal of drug metabolism and pharmacokinetics 43, 251-257

Lane, J. D., Steege, J. F., Rupp, S. L., and Kuhn, C. M. (1992). Menstrual cycle effects on caffeine elimination in the human female. European journal of clinical pharmacology 43, 543-6

Lane, S. D., Green, C. E., Schmitz, J. M., Rathnayaka, N., Fang, W. B., Ferré, S., et al. (2014). Comparison of caffeine and d-amphetamine in cocaine-dependent subjects: Differential outcomes on subjective and cardiovascular effects, reward learning, and salivary paraxanthine. Journal of addiction research \& therapy 5,176

Lelo, A., Birkett, D. J., Robson, R. A., and Miners, J. O. (1986a). Comparative pharmacokinetics of caffeine and its primary demethylated metabolites paraxanthine, theobromine and theophylline in man. British journal of clinical pharmacology 22, 177-82

Lelo, A., Miners, J. O., Robson, R. A., and Birkett, D. J. (1986b). Quantitative assessment of caffeine partial clearances in man. British journal of clinical pharmacology 22, 183-186. doi:10.1111/j. 1365-2125.1986.tb05247.x

Levy, M. and Zylber-Katz, E. (1983). Caffeine metabolism and coffee-attributed sleep disturbances. Clinical pharmacology and therapeutics 33, 770-5

Magnusson, M. O., Dahl, M.-L., Cederberg, J., Karlsson, M. O., and Sandström, R. (2008). Pharmacodynamics of carbamazepine-mediated induction of cyp3a4, cyp1a2, and pgp as assessed by probe substrates midazolam, caffeine, and digoxin. Clinical pharmacology and therapeutics 84, 52-62

Marks, V. and Kelly, J. F. (1973). Absorption of caffeine from tea, coffee, and coca cola. Lancet (London, England) 1, 827

Matthaei, J., Tzvetkov, M. V., Strube, J., Sehrt, D., Sachse-Seeboth, C., Hjelmborg, J. B., et al. (2016). Heritability of caffeine metabolism: Environmental effects masking genetic effects on cyp1a2 activity but not on nat2. Clinical pharmacology and therapeutics 100, 606-616

May, D. C., Jarboe, C. H., VanBakel, A. B., and Williams, W. M. (1982). Effects of cimetidine on caffeine disposition in smokers and nonsmokers. Clinical pharmacology and therapeutics 31, 656-61

McDonagh, J. E., Nathan, V. V., Bonavia, I. C., Moyle, G. R., and Tanner, A. R. (1991). Caffeine clearance by enzyme multiplied immunoassay technique: a simple, inexpensive, and useful indicator of liver function. Gut 32, 681-684. doi:10.1136/gut.32.6.681

McLean, C. and Graham, T. E. (2002). Effects of exercise and thermal stress on caffeine pharmacokinetics in men and eumenorrheic women. Journal of applied physiology (Bethesda, Md. : 1985) 93, 1471-8

McQuilkin, S. H., Nierenberg, D. W., and Bresnick, E. (1995). Analysis of within-subject variation of caffeine metabolism when used to determine cytochrome p4501a2 and n-acetyltransferase-2 activities. Cancer epidemiology, biomarkers \& prevention : a publication of the American Association for Cancer Research, cosponsored by the American Society of Preventive Oncology 4, 139-46

Millard, J. T., Passang, T., Ye, J., Kline, G. M., Beachy, T. M., Hepburn, V. L., et al. (2018). Genotype and phenotype of caffeine metabolism: A biochemistry laboratory experiment. Journal of Chemical Education 95, 1856-1860. doi:10.1021/acs.jchemed.8b00318

Miners, J. O. and Birkett, D. J. (1996). The use of caffeine as a metabolic probe for human drug metabolizing enzymes. General pharmacology 27, 245-249. doi:10.1016/0306-3623(95)02014-4

Mitchell, D. C., Knight, C. A., Hockenberry, J., Teplansky, R., and Hartman, T. J. (2014). Beverage caffeine intakes in the u.s. Food and chemical toxicology : an international journal published for the British Industrial Biological Research Association 63, 136-42 
Murphy, T. L., McIvor, C., Yap, A., Cooksley, W. G., Halliday, J. W., and Powell, L. W. (1988). The effect of smoking on caffeine elimination: implications for its use as a semiquantitative test of liver function. Clinical and experimental pharmacology \& physiology 15, 9-13

Muscat, J. E., Pittman, B., Kleinman, W., Lazarus, P., Stellman, S. D., and Richie, J. P. (2008). Comparison of cyp1a2 and nat2 phenotypes between black and white smokers. Biochemical pharmacology 76, 929-937. doi:10.1016/j.bcp.2008.07.024

Myrand, S. P., Sekiguchi, K., Man, M. Z., Lin, X., Tzeng, R.-Y., Teng, C.-H., et al. (2008). Pharmacokinetics/genotype associations for major cytochrome p450 enzymes in native and first- and third-generation japanese populations: comparison with korean, chinese, and caucasian populations. Clinical pharmacology and therapeutics 84, 347-361. doi:10.1038/sj.clpt.6100482

Nakazawa, K. and Tanaka, H. (1988). [pharmacokinetics of caffeine and dimethylxanthines in plasma and saliva]. Yakugaku zasshi : Journal of the Pharmaceutical Society of Japan 108, 653-8

Nehlig, A. (2018). Interindividual differences in caffeine metabolism and factors driving caffeine consumption. Pharmacological reviews 70, 384-411. doi:10.1124/pr.117.014407

Newton, R., Broughton, L. J., Lind, M. J., Morrison, P. J., Rogers, H. J., and Bradbrook, I. D. (1981). Plasma and salivary pharmacokinetics of caffeine in man. European journal of clinical pharmacology $21,45-52$

Oh, K.-S., Park, S.-J., Shinde, D. D., Shin, J.-G., and Kim, D.-H. (2012). High-sensitivity liquid chromatography-tandem mass spectrometry for the simultaneous determination of five drugs and their cytochrome p450-specific probe metabolites in human plasma. Journal of chromatography. B, Analytical technologies in the biomedical and life sciences 895-896, 56-64

Park, G. J.-H., Katelaris, P. H., Jones, D. B., Seow, F., Le Couteur, D. G., and Ngu, M. C. (2003). Validity of the 13c-caffeine breath test as a noninvasive, quantitative test of liver function. Hepatology (Baltimore, Md.) 38, 1227-36

Parsons, W. D. and Neims, A. H. (1978). Effect of smoking on caffeine clearance. Clinical pharmacology and therapeutics $24,40-5$

Patwardhan, R. V., Desmond, P. V., Johnson, R. F., and Schenker, S. (1980). Impaired elimination of caffeine by oral contraceptive steroids. The Journal of laboratory and clinical medicine 95, 603-8. PIP: TJ: JOURNAL OF LABORATORY AND CLINICAL MEDICINE.

Perera, V., Gross, A. S., and McLachlan, A. J. (2010). Caffeine and paraxanthine hplc assay for cyp1a2 phenotype assessment using saliva and plasma. Biomedical chromatography : BMC 24, 1136-44

Perera, V., Gross, A. S., Xu, H., and McLachlan, A. J. (2011). Pharmacokinetics of caffeine in plasma and saliva, and the influence of caffeine abstinence on cyp1a2 metrics. The Journal of pharmacy and pharmacology 63, 1161-8

Prescott, L. F., Critchley, J. A., Balali-Mood, M., and Pentland, B. (1981). Effects of microsomal enzyme induction on paracetamol metabolism in man. British journal of clinical pharmacology 12, 149-53

Prescott, L. F., Yoovathaworn, K., Makarananda, K., Saivises, R., and Sriwatanakul, K. (1993). Impaired absorption of paracetamol in vegetarians. British journal of clinical pharmacology 36, 237-40

Priller, S. (2005). Cytochrome p450 1a2 phenotyping for student laboratories. Pharmacy Education journal

Puri, B. K., Heard, C. R., and Monro, J. A. (2020). Is there a sex difference in adult salivary clearance of caffeine (1,3,7-trimethylpurine-2,6-dione)? Journal of oral biology and craniofacial research 10, 20-22. doi:10.1016/j.jobcr.2020.01.010

Rasmussen, B. B., Brix, T. H., Kyvik, K. O., and Brøsen, K. (2002). The interindividual differences in the 3-demthylation of caffeine alias cyp1a2 is determined by both genetic and environmental factors. 
Pharmacogenetics 12, 473-478. doi:10.1097/00008571-200208000-00008

Renner, B., Clarke, G., Grattan, T., Beisel, A., Mueller, C., Werner, U., et al. (2007). Caffeine accelerates absorption and enhances the analgesic effect of acetaminophen. Journal of clinical pharmacology 47, $715-26$

Renner, E., Wietholtz, H., Huguenin, P., Arnaud, M. J., and Preisig, R. (1984). Caffeine: a model compound for measuring liver function. Hepatology (Baltimore, Md.) 4, 38-46

Rietveld, E. C., Broekman, M. M., Houben, J. J., Eskes, T. K., and van Rossum, J. M. (1984). Rapid onset of an increase in caffeine residence time in young women due to oral contraceptive steroids. European journal of clinical pharmacology 26, 371-3. PIP: TJ: EUROPEAN JOURNAL OF CLINICAL PHARMACOLOGY.

Rubin, T. M., Heyne, K., Luchterhand, A., Bednarsch, J., W R Vondran, F., Polychronidis, G., et al. (2017). Kinetic validation of the limax test during 10000 intravenous , javax.xml.bind.jaxbelement@10fb8f50, c-methacetin breath tests. Journal of breath research 12, 016005. doi:10.1088/1752-7163/aa820b

Sadek, P., Pan, X., Shepherd, P., Malandain, E., Carney, J., and Coleman, H. (2017). A randomized, twoway crossover study to evaluate the pharmacokinetics of caffeine delivered using caffeinated chewing gum versus a marketed caffeinated beverage in healthy adult volunteers. Journal of caffeine research 7, 125-132

Schmider, J., Brockmöller, J., Arold, G., Bauer, S., and Roots, I. (1999). Simultaneous assessment of cyp3a4 and cyp1a2 activity in vivo with alprazolam and caffeine. Pharmacogenetics $9,725-34$

Schrenk, D., Brockmeier, D., Mörike, K., Bock, K. W., and Eichelbaum, M. (1998). A distribution study of cyp1a2 phenotypes among smokers and non-smokers in a cohort of healthy caucasian volunteers. European journal of clinical pharmacology 53, 361-367. doi:10.1007/s002280050394

Scott, N. R., Chakraborty, J., and Marks, V. (1984). Determination of caffeine, theophylline and theobromine in serum and saliva using high-performance liquid chromatography. Annals of clinical biochemistry 21 ( Pt 2), 120-124. doi:10.1177/000456328402100208

Scott, N. R., Stambuk, D., Chakraborty, J., Marks, V., and Morgan, M. Y. (1988). Caffeine clearance and biotransformation in patients with chronic liver disease. Clinical science (London, England : 1979) 74, 377-84

Scott, N. R., Stambuk, D., Chakraborty, J., Marks, V., and Morgan, M. Y. (1989). The pharmacokinetics of caffeine and its dimethylxanthine metabolites in patients with chronic liver disease. British journal of clinical pharmacology 27, 205-13

Seng, K.-Y., Fun, C.-Y., Law, Y.-L., Lim, W.-M., Fan, W., and Lim, C.-L. (2009). Population pharmacokinetics of caffeine in healthy male adults using mixed-effects models. Journal of clinical pharmacy and therapeutics 34, 103-14

Soto, J., Alsar, M. J., and Sacristan, J. A. (1995). Assessment of the time course of drugs with inhibitory effects on hepatic metabolic activity using successive salivary caffeine tests. Pharmacotherapy 15, 781-784

Spigset, O., Hägg, S., Söderström, E., and Dahlqvist, R. (1999). The paraxanthine:caffeine ratio in serum or in saliva as a measure of cyp1a2 activity: when should the sample be obtained? Pharmacogenetics 9, 409-12

Stader, F., Siccardi, M., Battegay, M., Kinvig, H., Penny, M. A., and Marzolini, C. (2019). Repository describing an aging population to inform physiologically based pharmacokinetic models considering anatomical, physiological, and biological age-dependent changes. Clinical pharmacokinetics 58, 483501. doi:10.1007/s40262-018-0709-7 
Stille, W., Harder, S., Mieke, S., Beer, C., Shah, P. M., Frech, K., et al. (1987). Decrease of caffeine elimination in man during co-administration of 4-quinolones. The Journal of antimicrobial chemotherapy 20, 729-34

Sudsakorn, S., Bahadduri, P., Fretland, J., and Lu, C. (2020). 2020 fda drug-drug interaction guidance: A comparison analysis and action plan by pharmaceutical industrial scientists. Current drug metabolism 21, 403-426. doi:10.2174/1389200221666200620210522

Syed, S. A., Kamimori, G. H., Kelly, W., and Eddington, N. D. (2005). Multiple dose pharmacokinetics of caffeine administered in chewing gum to normal healthy volunteers. Biopharmaceutics \& drug disposition 26, 403-9

Tamminga, W. J., Wemer, J., Oosterhuis, B., de Zeeuw, R. A., de Leij, L. F., and Jonkman, J. H. (2001). The prevalence of cyp2d6 and cyp2c19 genotypes in a population of healthy dutch volunteers. European journal of clinical pharmacology 57, 717-722. doi:10.1007/s002280100359

Tanaka, E., Ishikawa, A., Yamamoto, Y., Osada, A., Tsuji, K., Fukao, K., et al. (1993). Comparison of hepatic drug-oxidizing activity after simultaneous administration of two probe drugs, caffeine and trimethadione, to human subjects. Pharmacology \& toxicology 72, 31-3

Tanaka, S., Uchida, S., Inui, N., Takeuchi, K., Watanabe, H., and Namiki, N. (2014). Simultaneous lc-ms/ms analysis of the plasma concentrations of a cocktail of 5 cytochrome p450 substrate drugs and their metabolites. Biological \& pharmaceutical bulletin 37, 18-25

Tang-Liu, D. D., Williams, R. L., and Riegelman, S. (1983). Disposition of caffeine and its metabolites in man. The Journal of pharmacology and experimental therapeutics 224, 180-5

Tantcheva-Poór, I., Zaigler, M., Rietbrock, S., and Fuhr, U. (1999). Estimation of cytochrome p-450 cyp1a2 activity in 863 healthy caucasians using a saliva-based caffeine test. Pharmacogenetics 9, 131-144

Thacker, S. B. (1988). Meta-analysis. a quantitative approach to research integration. JAMA 259, 16851689. doi:10.1001/jama.259.11.1685

Tian, D.-D., Natesan, S., White, J., John R, and Paine, M. F. (2019). Effects of common cyp1a2 genotypes and other key factors on intraindividual variation in the caffeine metabolic ratio: An exploratory analysis. Clinical and translational science 12, 39-46

Trang, J. M., Blanchard, J., Conrad, K. A., and Harrison, G. G. (1982). The effect of vitamin c on the pharmacokinetics of caffeine in elderly men. The American journal of clinical nutrition 35, 487-94

Trang, J. M., Blanchard, J., Conrad, K. A., and Harrison, G. G. (1985). Relationship between total body clearance of caffeine and urine flow rate in elderly men. Biopharmaceutics \& drug disposition 6, 51-6

Tripathi, A., Tiwari, B., Patil, R., Khanna, V., and Singh, V. (2015). The role of salivary caffeine clearance in the diagnosis of chronic liver disease. Journal of oral biology and craniofacial research 5, 28-33

Turpault, S., Brian, W., Van Horn, R., Santoni, A., Poitiers, F., Donazzolo, Y., et al. (2009). Pharmacokinetic assessment of a five-probe cocktail for cyps 1a2, 2c9, 2c19, 2d6 and 3a. British journal of clinical pharmacology 68, 928-35

Urry, E., Jetter, A., and Landolt, H.-P. (2016). Assessment of cyp1a2 enzyme activity in relation to type-2 diabetes and habitual caffeine intake. Nutrition \& metabolism 13, 66. doi:10.1186/s12986-016-0126-6

Wahlländer, A., Mohr, S., and Paumgartner, G. (1990). Assessment of hepatic function. comparison of caffeine clearance in serum and saliva during the day and at night. Journal of hepatology 10, 129-137. doi:10.1016/0168-8278(90)90041-o

Walther, H., Banditt, P., and Köhler, E. (1983). [significance of caffeine values in serum, saliva and urinedetermination of pharmacokinetic data by non-invasive methods in psychopharmacologic studies]. Pharmacopsychiatria 16, 166-170. doi:10.1055/s-2007-1019492 
Wang, T., Kleber, G., Stellaard, F., and Paumgartner, G. (1985). Caffeine elimination: a test of liver function. Klinische Wochenschrift 63, 1124-8

White, J. R. J., Padowski, J. M., Zhong, Y., Chen, G., Luo, S., Lazarus, P., et al. (2016). Pharmacokinetic analysis and comparison of caffeine administered rapidly or slowly in coffee chilled or hot versus chilled energy drink in healthy young adults. Clinical toxicology (Philadelphia, Pa.) 54, 308-12

Yamazaki, T., Desai, A., Goldwater, R., Han, D., Howieson, C., Akhtar, S., et al. (2017). Pharmacokinetic effects of isavuconazole coadministration with the cytochrome p450 enzyme substrates bupropion, repaglinide, caffeine, dextromethorphan, and methadone in healthy subjects. Clinical pharmacology in drug development 6, 54-65. doi:10.1002/cpdd.281

Yang, X., Zhang, B., Molony, C., Chudin, E., Hao, K., Zhu, J., et al. (2010). Systematic genetic and genomic analysis of cytochrome p450 enzyme activities in human liver. Genome research 20, 1020 1036. doi:10.1101/gr.103341.109

Yesair, D. W., Branfman, A. R., and Callahan, M. M. (1984). Human disposition and some biochemical aspects of methylxanthines. Progress in clinical and biological research 158, 215-233

Yubero-Lahoz, S., Pardo, R., Farre, M., Mathuna, B. O., Torrens, M., Mustata, C., et al. (2012). Changes in cyp1a2 activity in humans after 3,4-methylenedioxymethamphetamine (mdma, ecstasy) administration using caffeine as a probe drug. Drug metabolism and pharmacokinetics 27, 605-13

Zanger, U. M. and Schwab, M. (2013). Cytochrome p450 enzymes in drug metabolism: regulation of gene expression, enzyme activities, and impact of genetic variation. Pharmacology \& therapeutics 138, 103-141. doi:10.1016/j.pharmthera.2012.12.007

Zylber-Katz, E., Granit, L., and Levy, M. (1984). Relationship between caffeine concentrations in plasma and saliva. Clinical pharmacology and therapeutics 36, 133-7 